\title{
Optical image processing in second-harmonic generation
}

\author{
Pierre Scotto $^{1}$, Pere Colet $^{1}$, Adrian Jacobo ${ }^{1}$, and Maxi San Miguel ${ }^{1}$ \\ Instituto Mediterraneo de Estudios Avanzados, IMEDEA (CSIC-UIB), Campus \\ Universitat de les Illes Balears, E-07122 Palma de Mallorca, Spain \\ pere@imedea.uib.es
}

\section{Introduction}

Although the processing of an image by all-optical means is quite less common than the well-developed techniques for digital image processing [1], it has nevertheless been around for quite a some time. At a classical level early works demonstrated frequency transfer of an optical image from the infrared to the visible domain $[2,3]$, and later from the visible to the UV domain $[4,5]$, as well as parametric amplification of an UV image [6,7], and contrast inversion [8]. In these schemes, an optical image at a frequency $\omega$ is directly injected into a nonlinear crystal illuminated with a strong monochromatic pump wave at frequency $\omega_{p}$ and the processed image is formed in the output plane. As a result of the nonlinearity of the crystal, the input image will be, depending on some phase matching condition, either transferred to a higher frequency $\omega+\omega_{p}$ by simple frequency addition $[2,3,4]$, or amplified by photon down conversion $[6,7,8]$. In the latter case the amplification is accompanied by the formation of a phase conjugated (idler) image at the complementary frequency $\omega_{p}-\omega$. Considering the spatial dependence of the image processing mechanism on the position of the object in the transverse plane, the phase matching condition will determine whether image processing will be efficient either on a disk centered on the optical main axis of the system, or on a ring of finite width. This latter regime is also useful for selectively amplifying some Fourier components of a given image, leading to contrast enhancement or inversion. A quite significant amount of work in all-optical image processing operations have been performed in photorefractive media [9] including edge enhancement $[10,11,12]$, image inversion, division, differentiation and deblurring $[13,14,15,16]$, noise suppression [17] and contrast enhancement [18].

More recently, image processing has been considered also on a quantum level, including the investigation of the properties of the quantum fluctuations in the output image. The crucial prediction, which gave raise to rapid developments in the emerging field of "quantum imaging" [19, 20], was made in the context of image amplification: whereas quantum mechanics imposes that the phase-insensitive amplification of an image is always accompanied by a addition of at least $3 \mathrm{~dB}$ extra noise to the output image [21], a phase sensitive amplifier has a much better noise performance [22, 23]. Even more, 
noiseless image amplification, i.e. an amplification which preserves the signalto-noise ratio during processing, was shown, first theoretically $[24,25]$, and then experimentally [26], to be possible. This technique may be applicable to situations, in which a faint coherent signal must be amplified prior to detection. In the case of weak signals, the degradation of the signal to noise ratio predicted by quantum mechanics in the case of phase insensitive amplification might irremediably destroy the information encoded in the image. Most of the quantum states,

In this chapter we consider the use of second harmonic generation for alloptical processing of images. From the point of view of the crystal nonlinearities one distinguishes between type-I and type II. second harmonic generation . In the simplest situation, type I refers to the case where two fields with the same polarization and the same fundamental frequency $\omega$ combine to yield a second harmonic field at frequency $(2 \omega)$. In type-II two linearly orthogonally polarized fields with fundamental frequency $\omega$ are injected in the nonlinear crystal leading to a second harmonic field at frequency $2 \omega$.

On one hand here we will address from a classical point of view the situation in which the nonlinear crystals is placed inside a optical cavity rather in the more common propagation configuration. The fundamental difference is the existence of instability thresholds which, if used appropriately, allow for a nonlinear processing of the image. For example, considering intracavity type-II second harmonic generation inside planar cavity where all the fields are resonant, it is possible to selectively enhance the contrast of part of an image or to detect its contour [27]. This will be discussed in section 2. On the other hand in sections 3 and 4 we will consider the quantum imaging properties of second harmonic generation in the propagation configuration. The first of these sections is devoted to type-I, while the second one is devoted to type-II where the polarization degree of freedom allows for a larger variety of possible operations.

\section{Image processing in second harmonic generation at a classical level}

In this section we consider a crystal with a $\chi^{(2)}$ nonlinearity enclosed in an optical cavity, taken ideally to be a planar cavity and we will assume type II phase matching as sketched in Fig 1. A second-harmonic (SH) field will be generated if the cavity is pumped at two orthogonal polarizations $x$ and $y$. In the paraxial and mean field approximation the system can be described by the following set of equations, [27, 28, 29, 30,31]

$$
\begin{aligned}
\partial_{t} B & =-\left(1+\imath \delta_{B}\right) B+\frac{\imath}{2} \nabla_{\perp}^{2} B+\imath A_{x} A_{y} \\
\partial_{t} A_{x} & =-\left(1+\imath \delta_{A}\right) A_{x}+\imath \nabla_{\perp}^{2} A_{x}-\imath A_{y}^{*} B+E_{x} \\
\partial_{t} A_{y} & =-\left(1+\imath \delta_{A}\right) A_{y}+\imath \nabla_{\perp}^{2} A_{y}-\imath A_{x}^{*} B+E_{y}
\end{aligned}
$$


which govern the temporal evolution of the intracavity field envelopes $A_{x}$ and $A_{y}$ at the fundamental frequency $\omega$ with linear polarization $x$ and $y$ and $B$ at the second harmonic frequency $2 \omega$, polarized along the $y$ axis. $\delta_{A}$ and $\delta_{B}$ are the detunings at the fundamental and second harmonic frequencies respectively. Times are expressed in units of the cavity decay time and lengths in units of the diffraction length and the diffraction is taken in account thorough the transverse Laplacian $\nabla_{\perp}^{2}$. The pumping amplitudes $E_{x}$ and $E_{y}$ in each linear polarization state are chosen such that an image is injected with the $x$ polarization while an homogeneous field is inserted in the $y$ polarization.

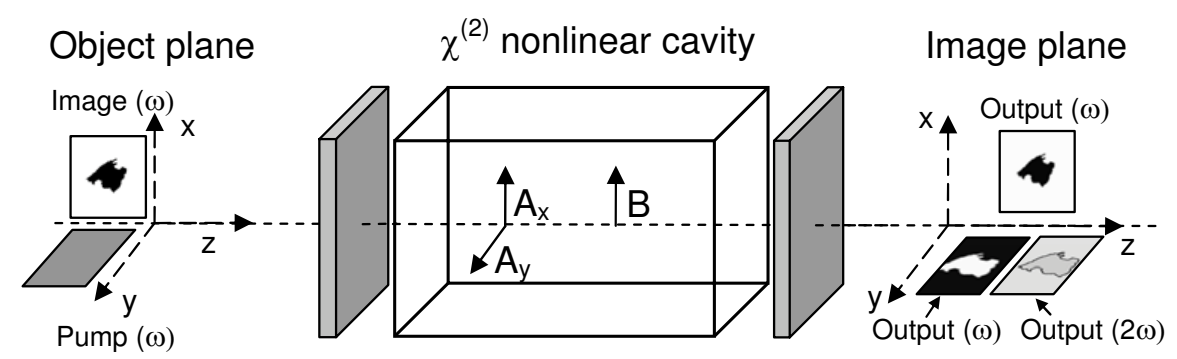

Fig. 1. Scheme of an optical device based on intracavity type-II second harmonic generation. The nonlinear crystal is enclosed in a plane mirror cavity.
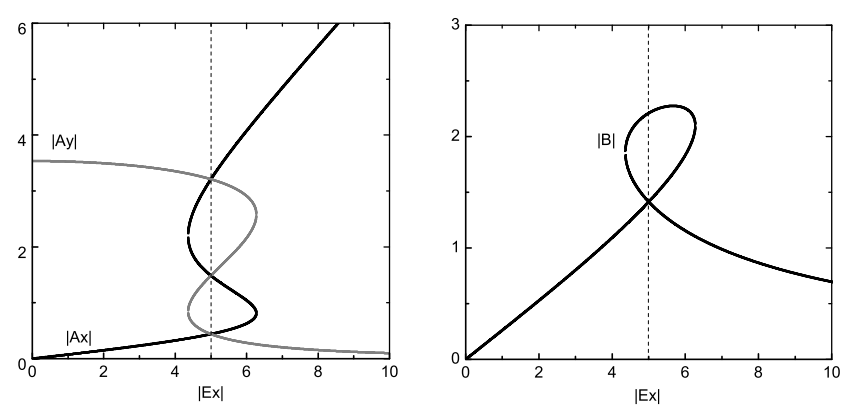

Fig. 2. Second harmonic generation for asymmetric pumping. Steady state intracavity field amplitudes as a function of $E_{x}$, for $E_{y}=5, \delta_{A}=1$ and $\delta_{B}=0$. The vertical dashed line corresponds to the symmetric pumping $E_{x}=E_{y}$

The study of the steady-state solution of Eqs.(1)-(3) for homogeneous pumps provides valuable insight into the relevant properties of the system that will be used for image processing. For pumps homogeneous in the transverse plane, $E_{x}$ and $E_{y}$ can be taken as real fields without loss of generality. Typically, Eqs. (1)-(3) have been considered for the case of symmetrical pumping $E_{x}=E_{y}$, which maximizes the production of second harmonic. In 
such case the homogeneous stationary steady state becomes unstable for a pump above the critical value $[28,29,30,31,32]$ :

$$
\left|E_{a s}\right|^{2}=2\left(1+\delta_{B}^{2}\right)^{1 / 2}\left(1+\delta_{A}^{2}\right)^{3 / 2}+2\left(1+\delta_{A}^{2}\right)\left(1-\delta_{A} \delta_{B}\right) .
$$

The system evolves to an homogeneous state for which $\left|A_{x}\right|$ and $\left|A_{y}\right|$ are different, so the intracavity field polarization is no longer the same as the pump (polarization instability). Because of the symmetry of the system two equivalent but different states can exist, one with a large value of $\left|A_{x}\right|$ and a low value of $\left|A_{y}\right|$ and the otherwise one [27, 28, 29].

For asymmetric homogeneous pumping $E_{x} \neq E_{y}$ the homogeneous steady state for $\left|A_{y}\right|$ is given by the solution of the polynomial

$$
\begin{aligned}
\Delta_{A}\left|A_{y}\right|^{10} & +\left[4\left(1-\delta_{b}\right) \Delta_{A}-\left|E_{y}\right|^{2}\right]\left|A_{y}\right|^{8} \\
& +2\left[\Delta_{A} Q+\Delta_{A B}\left(\left|E_{x}\right|^{2}-2\left|E_{y}\right|^{2}\right)\right]\left|A_{y}\right|^{6} \\
& +2\left[2 \Delta_{A}^{2} \Delta_{B}^{2} \Delta_{A B}-Q\left|E_{y}\right|^{2}-2 \Delta_{A B}^{2}\left|E_{x}\right|^{2}\right]\left|A_{y}\right|^{4} \\
& +\left[\Delta_{A}^{3} \Delta_{B}^{2}+2 \Delta_{A} \Delta_{B} \Delta_{A B}\left(\left|E_{x}\right|^{2}-2\left|E_{y}\right|^{2}\right)\right]\left|A_{y}\right|^{2} \\
& -\Delta_{A}^{2} \Delta_{B}^{2}\left|E_{y}\right|^{2}=0 .
\end{aligned}
$$

where $\Delta_{A}=1+\delta_{A}^{2}, \Delta_{B}=1+\delta_{B}^{2}, \Delta_{A B}=1-\delta_{A} \delta_{B}$ and $Q=\left(\delta_{A}+\delta_{B}\right)^{2}+$ $3 \Delta_{A B}^{2}$. Once $\left|A_{y}\right|$ is known, $\left|A_{x}\right|$ and $|B|$ are given by

$$
\begin{aligned}
\left|A_{x}\right|^{2} & =\frac{\Delta_{B}\left|E_{x}\right|^{2}}{\left|A_{y}\right|^{2}+2 \Delta_{A B}\left|A_{y}\right|+\Delta_{A} \Delta_{B}} \\
|B|^{2} & =\frac{\left|A_{x}\right|^{2}\left|A_{y}\right|^{2}}{\Delta_{B}}
\end{aligned}
$$

Fig.2 shows the typical dependence of the stationary solutions for the intracavity fields $\left|A_{x}\right|,\left|A_{y}\right|$ and $|B|$ on $\left|E_{x}\right|$ when $E_{y}=5$. For small $\left|E_{x}\right|$, the functions $A_{x}\left(E_{x}\right)$ and $B\left(E_{x}\right)$ take small values while $A_{y}\left(E_{x}\right)$ is large and close to $E_{y} /\left(1+i \delta_{A}\right)$. All of them are single valued. When $\left|E_{x}\right|$ approaches $\left|E_{y}\right|$ the system displays bistability. $A_{x}\left(E_{x}\right)$ and $A_{y}\left(E_{x}\right)$ become $\mathrm{S}$ shaped and $B\left(E_{x}\right)$ closes over itself. For large $\left|E_{x}\right|$ all the functions become again single valued but now $A_{x}\left(E_{x}\right) \gg A_{y}\left(E_{x}\right)$. The existence of three steady-state solutions of Eqs. (1)-(3) in a region of finite width centered on $\left|E_{x}\right|=\left|E_{y}\right|$ is closely related to the polarization instability occurring in the symmetrical pumping case. If fact,this $\mathrm{S}$ shape can be observed only if $\left|E_{y}\right|>\left|E_{a s}\right|$

In the following we consider the effects produced on an image inserted in the system as spatial variations in the intensity of the $x$-polarized pump field along with an homogeneous pump $E_{y}$. Varying the amplitude of the homogeneous pump it is possible to achieve different regimes of operation. In a first regime the image can be transferred from the fundamental to the second harmonic. In a second regime it is possible to enhance its contrast and to detect the contour of the image [27]. Furthermore, it is also possible to filter noise eventually present in the image. For simplicity $E_{x}$ and $E_{y}$ are 
taken as real fields except when noise is considered. Here we will consider only the case of an ideal cavity with flat mirrors which is resonant with both fundamental fields and with the second harmonic field. Similar operations can be performed using cavities with spherical mirrors or where only the fundamental fields are resonant as discussed in Ref. [33].

\subsection{Frequency up-conversion of an image}

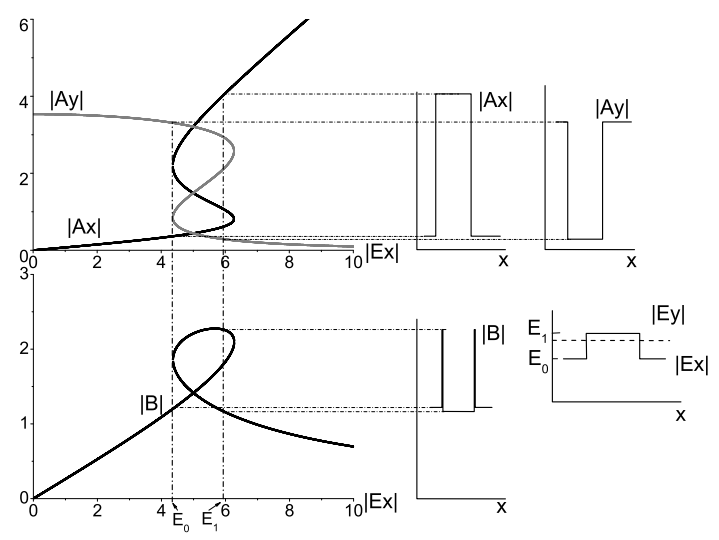

Fig. 3. Geometrical construction to illustrate the frequency transfer regime. On the left we plot the stationary amplitude of the intracavity fields for homogeneous asymmetric pumping as a function of $E_{x}$ for $E_{y}=5\left(\delta_{B}=0, \delta_{A}=1\right.$, and $\left.E_{a s}=3.10755\right)$. On the right we plot the response of the system to a simple image (sketched on the far right) where $E_{x}$ takes only the values $E_{0}$ and $E_{1}$ with $E_{0}<E_{1}<E_{y}$.

We consider the injection of an image, that is, the amplitude of the $\mathrm{x}$ polarized signal $\left|E_{x}(x)\right|$ is a function of the transverse coordinate $x$. At a given position $x$ the intracavity fields $A_{x, y}(x)$ and $B(x)$ tend to take the stationary values shown in Fig. 2 as if the pumps where homogeneous, despite of the spatial coupling caused by diffraction. Diffraction becomes relevant for image details on the scale of the diffraction length. Figure 3 shows an sketch for a very simple 1-dimensional image where $\left|E_{x}\right|$ takes only two values. If $\left|E_{x}(x)\right|$ remains well below $\left|E_{y}\right|, A_{x}(x)$ never leaves the lower branch of the curve $A_{x}\left(E_{x}\right)$, so $\left|A_{x}\right|$ reproduces the spatial distribution of the input image $\left|E_{x}\right|(x)$. The output at the SH frequency $B(x)$ also reproduces $\left|E_{x}(x)\right|$. Therefore intracavity type II SHG allows an input image to be transferred from the fundamental to the $\mathrm{SH}$ frequency. In addition, polarization switching is performed, since the image encoded in $\left|E_{x}\right|$ and the SH field $B$ have orthogonal polarizations. As a side effect, the input image appears in negative as a weak modulation of $A_{y}$ around $E_{y} /\left(1+\imath \delta_{A}\right)$. This procedure is 

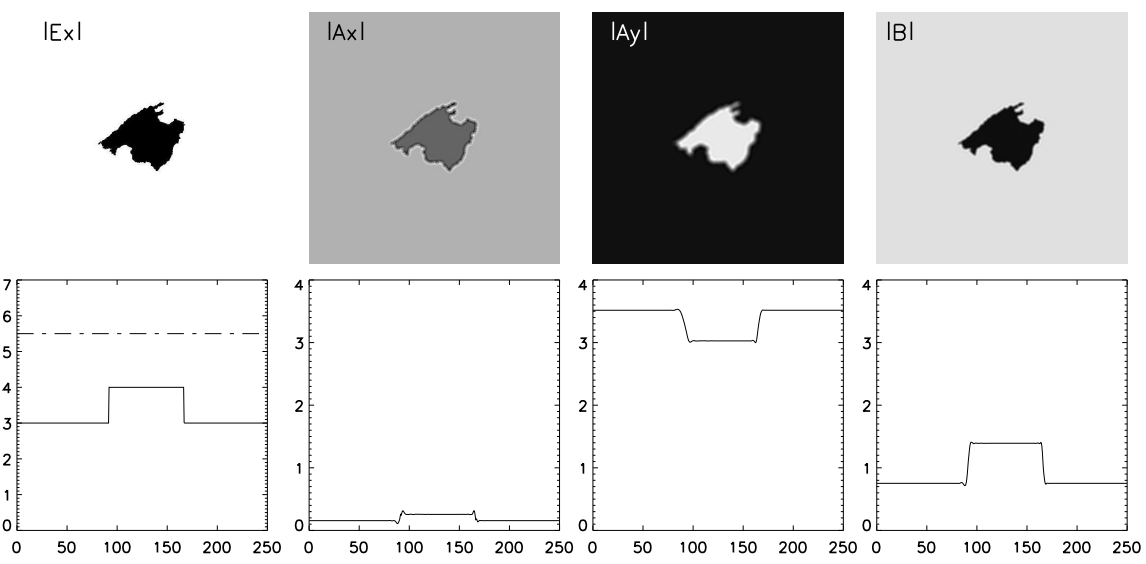

Fig. 4. Frequency transfer. The left column shows (from top to bottom) the spatial distribution of amplitude input field $E_{x}$, and the amplitude of the intracavity fields $\left|A_{x}\right|,\left|A_{y}\right|$ and $|B|$. In all the figures of the manuscript, except if otherwise noted, the grey scale of goes from the minimum (white) to the maximum (black) of each field. The right column shows a transversal cut of the fields along the dashed line on the top left panel. We have considered $E_{y}=5.5$ (shown as dashed line on the top right panel).

illustrated in Fig.4 in a more realistic two dimensional image. In this image can be seen that as an effect of the diffraction the edges of the image are soften. The image in the intracavity and second harmonic fields can be considered as the union of two different stationary states and the oscillatory tails of the front connecting the two states induce some distortion near the border. Nevertheless the image is quite well reproduced as seen in Fig.4.

\subsection{Contrast enhancement and contour recognition}

Now we will consider the case in which the amplitude of the signal locally exceeds $\left|E_{y}\right|$. In this case the multivalued dependence of $A_{x}\left(E_{x}\right), A_{y}\left(E_{x}\right)$ and $B\left(E_{x}\right)$ comes into play as is shown in Fig.5. If $E_{1}$ is larger than the upper end of the hysteresis cycle and $E_{0}$ is smaller than the lower end, $A_{x}\left(E_{x}(x)\right)$ has to jump from the lower to the upper branch, while $A_{y}\left(E_{x}(x)\right)$ has to jump from the upper to the lower branch. This will give rise to a sharp spatial variation of $A_{x}$ and $A_{y}$. In fact for vanishing intracavity fields as initial condition, it is not necessary to fully cross the hysteresis cycle to have a jump. With those initial conditions where $\left|E_{x}\right|<\left|E_{y}\right|$ the system locally selects the steady state solution with a small value for $\left|A_{x}\right|$ and a large value for $\left|A_{y}\right|$, while it selects the steady state with large $\left|A_{x}\right|$ and small $\left|A_{y}\right|$ where $\left|E_{x}\right|>\left|E_{y}\right|$. Therefore, the reference value $\left|E_{y}\right|$ plays in fact the role of an effective threshold and the jump already occurs if $\left|E_{x}\right|$ crosses $\left|E_{y}\right|$ as sketched in Fig.5. In the region where $\left|E_{x}(x)\right|$ is larger than 


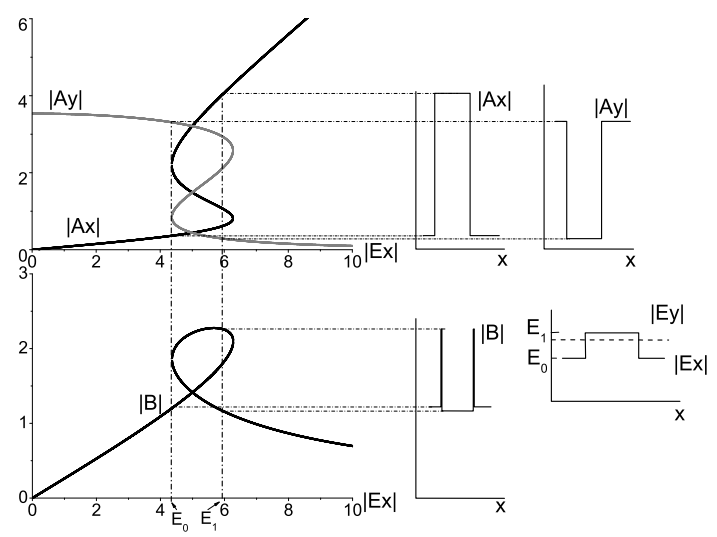

Fig. 5. Geometrical construction to illustrate the contrast enhancement and contour recognition regime similar to Fig. 3 but with $E_{0}<E_{y}<E_{1}$.

$|E x|$

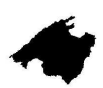

$|A x|$

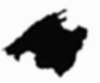

$\mid$ Ay
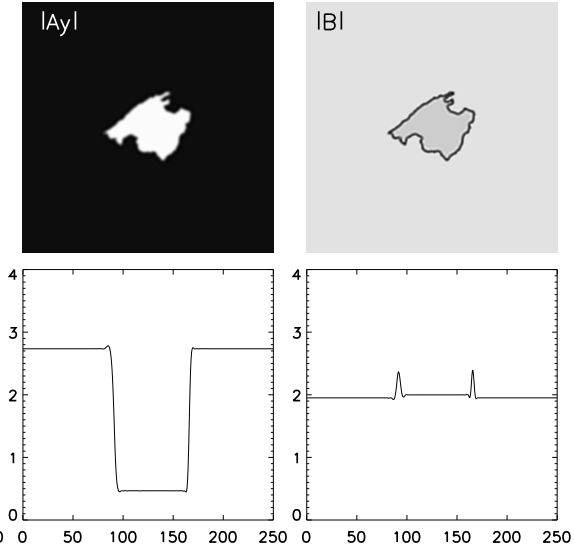

$|B|$
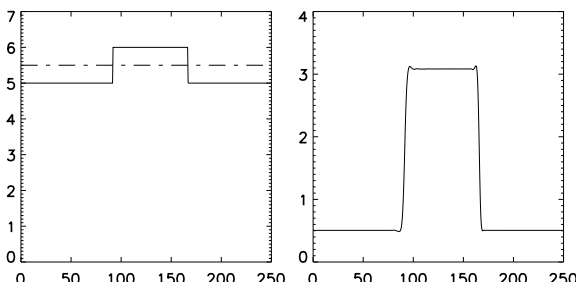

Fig. 6. Contrast enhancement and contour recognition. The left column shows (from top to bottom) the spatial distribution of amplitude of the input image $\left|E_{x}\right|$, and the amplitude of the intracavity fields $\left|A_{x}\right|,\left|A_{y}\right|$ and $|B|$. The gray scale of goes from the minimum (white) to the maximum (black) of each field. The right column shows a transversal cut of the fields. We have considered $\left|E_{y}\right|=5$.

the reference level $\left|E_{y}\right|,\left|A_{x}\right|$ has a large value compared with the zones where $\left|E_{x}(x)\right|<\left|E_{y}\right|$; so the contrast in this field appears enhanced with respect to the contrast in the input fields (Fig.5). The amplitude $\left|A_{y}\right|$ takes lower values where $\left|E_{x}\right|<\left|E_{y}\right|$ leading to an image which is inverted with respect to the input (Fig.5). At the border between the regions $\left|E_{x}\right|>\left|E_{y}\right|$ and $\left|E_{x}\right|<\left|E_{y}\right|$ the second harmonic field $B$ displays a sharp peak, since locally $\left|A_{x}\right| \simeq\left|A_{y}\right|$; i.e. the system goes trough the symmetric steady-sate solution characterized by a higher intracavity second harmonic field than the asymmetric stable ones. As a consequence the second harmonic field displays the contour of the 
input image Fig.5. These effects are shown for two dimensional input image in Fig.6. Image processing is slightly affected by diffraction effects in two dimensions, which tends to smooth out sharp angles in the input image and sets a minimum contrast below which no contrast enhancement can occur. It should be emphasized that the previous results show that for a given image different processing capabilities are possible tuning the amplitude of the homogeneous field $\left|E_{y}\right|$. This is even more interesting when considering images which are composed of many levels of intensity, as in a gray scale image. In that sense, if the homogeneous pump $\left|E_{y}\right|$ is set to a value larger than $\left|E_{x}(x)\right|$ for any $x$ then the frequency transfer process will take place and the whole image will be displayed by the second harmonic frequency field $B(x)$. If $\left|E_{y}\right|$ is decreased, then the parts of the image where $\left|E_{x}(x)\right|>\left|E_{y}\right|$ will undergo a contrast enhancement process.

\subsection{Noise filtering properties}
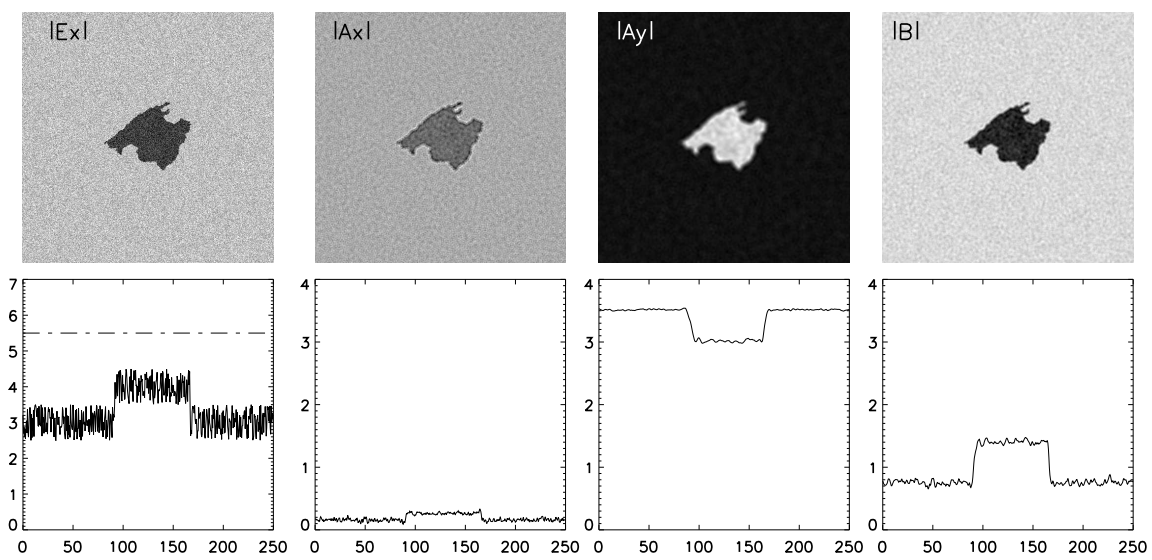

Fig. 7. Noise filtering in the frequency transfer regime. The left column shows (from top to bottom) the spatial distribution of amplitude of the input image $\left|E_{x}\right|$, and the amplitude of the intracavity fields $\left|A_{x}\right|,\left|A_{y}\right|$ and $|B|$. The gray scale of goes from the minimum (white) to the maximum (black) of each field. The right column shows a transversal cut of the fields. We have considered $\left|E_{y}\right|=5$.

Another interesting effect arises when the image inserted is superimposed with a complex random field, creating a noisy image both in intensity and phase. In this case the system shows noise filtering properties, and the images at the fundamental and second harmonic fields have a lower noise level than the input image. The noise filtering effect arises as an interplay between the diffraction and the nonlinear interaction which filters out all the high spatial frequency components of the input image are dissipated trough the 

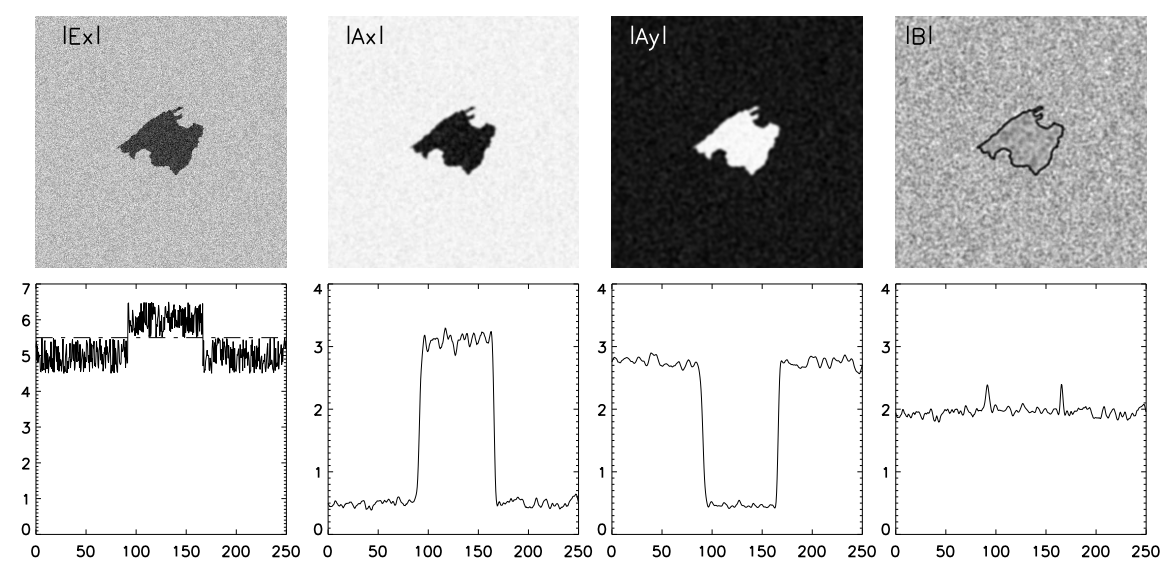

Fig. 8. Noise filtering in the contrast enhancement regime. The left column shows (from top to bottom) the spatial distribution of amplitude of the input image $\left|E_{x}\right|$, and the amplitude of the intracavity fields $\left|A_{x}\right|,\left|A_{y}\right|$ and $|B|$. The gray scale of goes from the minimum (white) to the maximum (black) of each field. The right column shows a transversal cut of the fields. We have considered $\left|E_{y}\right|=5.5$.

cavity losses. Therefore the small scale fluctuations associated to noise are effectively removed [27]. It appears both in the frequency transfer regime and in the contour recognition regime but it is more effective in the second case, when the nonlinearities play a more important role and the contrast of the image is enhanced, as can be seen from Figs. 7 and 8 .

\section{Quantum image processing in type-I second-harmonic generation}

The treatment in the previous section was fully classical. In this and the in the next section we will explore the possibilities offered by second harmonic generation for image processing from a quantum point of view. We will consider a traveling wave configuration since this case we will be able to separate the classically strong fields form the (weak) quantum fluctuations for which we will obtain linearized propagation equations. In this section we will consider the simpler case of a type-I interaction where the fundamental field has only one relevant polarization direction while in the next section we will address the type II case where the polarization degree of freedom of the fundamental fields play an important role. In the following subsections we address the dynamics of the field operators, obtain the propagation equations and explore its results for different configurations. 


\subsection{Field operator dynamics}

We follow a procedure similar to the one used in Ref. [25] for the OPA. The main difference that for an OPA it is generally justified to work in a classical approximation for the pump second-harmonic field since it is undepleted. In SHG, pump depletion cannot be neglected and both fundamental and second-harmonic field have to be treated simultaneously as quantum fields [34]. This section is divided in two parts, we first deduce the nonlinear propagation equations for the operators associated to the fundamental and second harmonic fields and then we linearize the quantum fluctuations around the nonlinear classical fields.

\section{A. Propagation equations}

We will begin by defining the slowly varying photon annihilation operators for the fundamental and second harmonic field $\mathcal{A}_{F}(z, \boldsymbol{\rho}, t)$ and $\mathcal{A}_{S}(z, \boldsymbol{\rho}, t)$ from the positive frequency part of the electric field,

$$
E_{i}^{(+)}(z, \boldsymbol{\rho}, t)=\imath \xi_{i} \sqrt{\frac{\hbar \omega_{i}}{2 \epsilon_{0} c}} \exp \left[\imath\left(k_{i} z-\omega_{i} t\right)\right] \mathcal{A}_{i}(z, \boldsymbol{\rho}, t),
$$

for $i=F, S$. The wave numbers of fundamental and second-harmonic waves in the nonlinear medium, $k_{F}$ and $k_{S}$, depend on the wave frequency trough the dispersion relation $\omega=\omega(k)$. In Eqs. (6) the prefactors

$$
\xi_{i}=\frac{u\left(k_{i}\right) v\left(k_{i}\right)}{c^{2} \cos \rho\left(k_{i}\right)}
$$

involve the group velocity $u\left(k_{i}\right)$, the phase velocity $v\left(k_{i}\right)$ and some generalized anisotropy angle $\rho\left(k_{i}\right)$. They describe the strength of the electric field in the medium, as compared to that in the vacuum. $z$ is the coordinate on the longitudinal axis, which is defined as the beam axis, and $\boldsymbol{\rho}$ is the two dimensional coordinate vector in the transverse plane.

The dynamics of these two fields in a $\chi^{(2)}$ nonlinear crystal are described by the Hamiltonian operator $[25,34]$ :

$$
\hat{H}_{i n t}=\hat{H}_{0, F}+\hat{H}_{0, S}+\hat{H}_{i n t}
$$

in which $\hat{H}_{0, F}$ and $\hat{H}_{0, S}$ are the free field Hamiltonians for the fundamental and second-harmonic field in the medium, whereas $\hat{H}_{\text {int }}$ describes the interaction between the two fields generated by the nonlinearity of the crystal. In terms of the slowly varying operators $\mathcal{A}_{i}(z, \boldsymbol{\rho}, t)$, the free-field Hamiltonians are given by [25]

$$
\hat{H}_{0, i}=\frac{\hbar \omega_{i}}{c} \int_{V} d z d^{2} \rho \mathcal{A}_{i}^{\dagger}(z, \boldsymbol{\rho}, t) \mathcal{A}_{i}(z, \boldsymbol{\rho}, t)
$$


where space integration is extended to the whole volume of the crystal. The expectation value $\left\langle\mathcal{A}_{i}^{\dagger}(z, \boldsymbol{\rho}, t) \mathcal{A}_{i}(z, \boldsymbol{\rho}, t)\right\rangle$ can be interpreted as the energy density per unit volume, scaled by a factor $\hbar \omega_{i} / c$. The interaction part $\hat{H}_{\text {int }}$ describes the three-wave interaction, which, under the usual assumption of a instantaneous and local nonlinear response of the medium [35], is given in terms of terms of the slowly varying field operators by

$$
\begin{aligned}
\hat{H}_{i n t}=\imath \hbar \lambda \int_{V} d z d^{2} \rho & {\left[e^{\imath \Delta k z} \mathcal{A}_{S}^{\dagger}(z, \boldsymbol{\rho}, t) \mathcal{A}_{F}^{2}(z, \boldsymbol{\rho}, t)\right.} \\
& \left.-e^{-\imath \Delta k z} \mathcal{A}_{S}(z, \boldsymbol{\rho}, t) \mathcal{A}_{F}^{\dagger 2}(z, \boldsymbol{\rho}, t)\right]
\end{aligned}
$$

where $\hbar \lambda=\chi^{(2)}\left(\hbar / 2 \epsilon_{0} c\right)^{3 / 2} \xi_{F}^{2} \xi_{S} \sqrt{\omega_{F}^{2} \omega_{S}}$ and $\Delta k=2 k_{F}-k_{S}$ is the collinear phase mismatch. $\hat{H}_{\text {int }}$ is the sum of two contributions: the first term in Eq.(10) is responsible for second-harmonic generation while the second for photon down-conversion. The dynamics of the two field operators are described by the Heisenberg equations, which for the Hamiltonian defined by Eqs. (9) and (10) are:

$$
\begin{aligned}
\partial_{t} \mathcal{A}_{F}(z, \boldsymbol{\rho}, t) & =\imath \omega_{F} \mathcal{A}_{F}(z, \boldsymbol{\rho}, t) \\
-\imath \omega_{F} & \int_{V} d z^{\prime} d^{2} \rho^{\prime} G_{F}\left(z-z^{\prime}, \boldsymbol{\rho}-\boldsymbol{\rho}^{\prime}\right) \mathcal{A}_{F}\left(z^{\prime}, \boldsymbol{\rho}^{\prime}, t\right) \\
-2 c \lambda & \int_{V} d z^{\prime} d^{2} \rho^{\prime} G_{F}\left(z-z^{\prime}, \boldsymbol{\rho}-\boldsymbol{\rho}^{\prime}\right) e^{-\imath \Delta k z^{\prime}} \mathcal{A}_{S}\left(z^{\prime}, \boldsymbol{\rho}^{\prime}, t\right) \mathcal{A}_{F}^{\dagger}\left(z^{\prime}, \boldsymbol{\rho}^{\prime}, t\right) \\
\partial_{t} \mathcal{A}_{S}(z, \boldsymbol{\rho}, t)= & \imath \omega_{S} \mathcal{A}_{S}(z, \boldsymbol{\rho}, t) \\
-\imath \omega_{S} & \int_{V} d z^{\prime} d^{2} \rho^{\prime} G_{S}\left(z-z^{\prime}, \boldsymbol{\rho}-\boldsymbol{\rho}^{\prime}\right) \mathcal{A}_{S}\left(z^{\prime}, \boldsymbol{\rho}^{\prime}, t\right) \\
-2 c \lambda & \int_{V} d z^{\prime} d^{2} \rho^{\prime} G_{F}\left(z-z^{\prime}, \boldsymbol{\rho}-\boldsymbol{\rho}^{\prime}\right) e^{-\imath \Delta k z^{\prime}} \mathcal{A}_{F}^{2}\left(z^{\prime}, \boldsymbol{\rho}^{\prime}, t\right)
\end{aligned}
$$

where

$$
G_{i}\left(z-z^{\prime}, \boldsymbol{\rho}-\boldsymbol{\rho}^{\prime}\right)=\int \frac{d k_{z} d^{2} q}{(2 \pi)^{3}} \frac{\omega\left(\sqrt{k_{z}^{2}+\boldsymbol{q}^{2}}\right)}{\omega_{i}} e^{\imath\left(k_{z}-k_{i}\right)\left(z-z^{\prime}\right)+\imath \boldsymbol{q}\left(\boldsymbol{\rho}-\boldsymbol{\rho}^{\prime}\right)} .
$$

It is helpful to work with operators in Fourier space rather than in real space:

$$
\mathcal{A}_{\sigma}(z, \boldsymbol{q}, \Omega)=\int d^{2} \rho e^{-\imath \boldsymbol{q} \cdot \boldsymbol{\rho}} \int d t e^{\imath \Omega \cdot t} \mathcal{A}_{\sigma}(z, \boldsymbol{\rho}, t)
$$

To separate the effects of free propagation through the crystal form the nonlinear effects is convenient to define for each field a propagation-corrected Fourier amplitude

$$
\hat{A}_{i}(z, \boldsymbol{q}, \Omega)=\xi_{i} \sqrt{n_{i}} \exp \left\{-\imath\left[k_{i}^{z}(\boldsymbol{q}, \Omega)-k_{i}\right] z\right\} \mathcal{A}_{i}(z, \boldsymbol{q}, \Omega)
$$

where $k_{i}^{z}(\boldsymbol{q}, \Omega)=\sqrt{k\left(\omega_{i}+\Omega\right)^{2}-\boldsymbol{q}^{2}}$, is the longitudinal wave number of a wave with frequency $\omega_{i}+\Omega$ and transverse vector $\boldsymbol{q}$. The exponential phase 
factor in Eq. (14) is chosen to absorb in the free propagation case the exact $z$ dependence of the wave associated to the field operator $\hat{A}_{i}(z, \boldsymbol{q}, \Omega)$. The prefactor $\xi_{i} \sqrt{n_{i}}=\sqrt{u_{i} / c}$, with $u_{i}$ defined as the group velocity of a wave with frequency $\omega_{i}$, allows to identify $\left\langle\hat{A}_{i}^{\dagger}(z, \boldsymbol{\rho}, t) \hat{A}_{i}(z, \boldsymbol{\rho}, t)\right\rangle$ with the mean photon flux density in the medium in (photons $/ \mathrm{cm}^{2} / \mathrm{sec}$ ).

In the standard paraxial $\left(|\boldsymbol{q}| \ll k_{\sigma}^{z}(\boldsymbol{q}, \Omega)\right)$ and quasimonochromatic $(\Omega \ll$ $\left.\omega_{\sigma}\right)$ approximation and under the assumption of slow $z$ dependence of the field operators, it can be shown that the propagation-corrected Fourier amplitudes obey the following set of propagation equations [34]:

$$
\begin{aligned}
& \frac{\partial}{\partial z} \hat{A}_{F}(z, \boldsymbol{q}, \Omega)=-2 K \int d^{2} q^{\prime} d \Omega^{\prime} \hat{A}_{F}^{\dagger}\left(z, \boldsymbol{q}^{\prime}, \Omega^{\prime}\right) \hat{A}_{S}\left(z, \boldsymbol{q}+\boldsymbol{q}^{\prime}, \Omega+\Omega^{\prime}\right) \\
& \times \exp \left\{\imath\left[k_{S}^{z}\left(\boldsymbol{q}+\boldsymbol{q}^{\prime}, \Omega+\Omega^{\prime}\right)-k_{F}^{z}(\boldsymbol{q}, \Omega)-k_{F}^{z}\left(\boldsymbol{q}^{\prime}, \Omega^{\prime}\right)\right] z\right\} \\
& \frac{\partial}{\partial z} \hat{A}_{S}(z, \boldsymbol{q}, \Omega)=+K \int d^{2} q^{\prime} d \Omega^{\prime} \hat{A}_{F}\left(z, \boldsymbol{q}^{\prime}, \Omega^{\prime}\right) \hat{A}_{F}\left(z, \boldsymbol{q}-\boldsymbol{q}^{\prime}, \Omega-\Omega^{\prime}\right) \\
& \times \exp \left\{\imath\left[k_{F}^{z}\left(\boldsymbol{q}^{\prime}, \Omega^{\prime}\right)+k_{F}^{z}\left(\boldsymbol{q}-\boldsymbol{q}^{\prime}, \Omega-\Omega^{\prime}\right)-k_{S}^{z}(\boldsymbol{q}, \Omega)\right] z\right\}
\end{aligned}
$$

where $K=(2 \pi)^{-3} \sqrt{c^{3} / u_{F}^{2} u_{S}} \lambda$ is the coupling constant of the interaction. These nonlinearly coupled differential-operator equations describe the propagation of the fundamental and second-harmonic field through the nonlinear medium. The right-hand side represent a sum over all three wave processes that are able to generate a fundamental and a second harmonic wave, respectively with transverse wavevector $\boldsymbol{q}$ and frequency $\Omega$ under the physical constraints of momentum and energy conservation. These equations generalize the propagation equations derived in [36] and [37] for a single mode case.

By solving Eqs. (16) and (17) one can obtain the functional dependence of the fields at the output plane with the fields at the input one. In principle, this allows to calculate the output of the system for an arbitrary quantummechanical state of the electromagnetic field illuminating the crystal. However, due to the nonlinear character of the equations some approximations are needed to solve them, as described bellow.

\section{B. Two-field input-output relations}

We consider now a situation suitable for image-processing problems: we assume the field distribution in the input plane of the crystal as given by the superposition of a strong homogeneous pump field at frequency $\omega$ and a weak cohrent signal at $2 \omega$ with some space-time distribution corresponding to an imput image. We consider that at any point inside the crystal the fundamental field generated by the imput signal remains weak with respect to the depleted pump field. Following [36] and [37] we write the propagationcorrected field operators associated with fundamental and second-harmonic field as 


$$
\begin{gathered}
\hat{A}_{F}(z \cdot \boldsymbol{q}, \Omega)=\tilde{c}_{F}(z) \delta^{(2)}(\boldsymbol{q}) \delta(\Omega)+\hat{a}_{F}(z, \boldsymbol{q}, \Omega) \\
\hat{A}_{S}(z \cdot \boldsymbol{q}, \Omega)=\tilde{c}_{S}(z) \delta^{(2)}(\boldsymbol{q}) \delta(\Omega)+\hat{a}_{S}(z, \boldsymbol{q}, \Omega)
\end{gathered}
$$

where $\tilde{c}_{F}(z)$ and $\tilde{c}_{S}(z)$ are the amplitudes of the strong monochromatic waves at frequencies $\omega$ and $2 \omega$ generated by the pump inside the crystal which, for simplification, are considered in the plane-wave approximation, and $\hat{a}_{F}(z, \boldsymbol{q}, \Omega)$ and $\hat{a}_{S}(z, \boldsymbol{q}, \Omega)$ are the quantum field operators associated with the two fields. These take into account the propagation of any field distribution injected into the crystal in addition to the strong pump field. In particular, they encode the propagation of the vacuum fluctuations entering the crystal trough the input plane, which are responsible for the quantum fluctuations in the output fields, as analyzed in [36, 37]. Substituting Eqs. (18) and (19) in Eqs. (16) and (17) we obtain at zero order

$$
\begin{aligned}
\frac{d}{d z} \tilde{c}_{F}(z) & =-2 K \tilde{c}_{F}^{*}(z) \tilde{c}_{S}(z) e^{-\imath \Delta k z} \\
\frac{d}{d z} \tilde{c}_{S}(z) & =K \tilde{c}_{F}^{2}(z) e^{\imath \Delta k z}
\end{aligned}
$$

which are the classical propagation equations of nonlinear optics. The total power $W$ is conserved $\left|\tilde{c}_{F}(z)\right|^{2}+2\left|\tilde{c}_{S}(z)\right|^{2}=\left|\tilde{c}_{F}(0)\right|^{2}+2\left|\tilde{c}_{S}(0)\right|^{2}=W$ in correspondence with the conservation of energy flow in the lossless crystal (Manley-Rowe relation). Introducing the dimensionless characteristic interaction length $z_{0}=1 / \sqrt{2 W} K$ and scaling the space as $\zeta=z / z_{0}$ and the field amplitudes as $c_{F}(z)=\tilde{c}_{F}(z) / \sqrt{W}$ and $c_{S}(z)=\tilde{c}_{S}(z) / \sqrt{W / 2}$ one gets

$$
\begin{aligned}
\frac{d}{d \zeta} c_{F}(\zeta) & =-c_{F}^{*}(\zeta) c_{S}(\zeta) e^{-\imath \Delta s \zeta} \\
\frac{d}{d \zeta} c_{S}(\zeta) & =c_{F}^{2}(\zeta) e^{\imath \Delta s \zeta},
\end{aligned}
$$

where $\Delta s=\Delta k z_{0}$. These equations can be solved analytically [47]. At first order one obtains

$$
\begin{aligned}
& \frac{\partial}{\partial \zeta} \hat{a}_{F}(\zeta, \boldsymbol{q}, \Omega)=-c_{S}(\zeta) \hat{a}_{F}^{\dagger}(\zeta,-\boldsymbol{q},-\Omega) e^{-\imath \Delta(\boldsymbol{q}, \Omega) \zeta} \\
& -\sqrt{2} c_{F}^{*}(\zeta) \hat{a}_{S}(\zeta, \boldsymbol{q}, \Omega) e^{\imath D(\boldsymbol{q}, \Omega) \zeta} \\
& \frac{\partial}{\partial \zeta} \hat{a}_{S}(\zeta, \boldsymbol{q}, \Omega)=\sqrt{2} c_{F}(\zeta) \hat{a}_{F}(\zeta, \boldsymbol{q}, \Omega) e^{\imath D(\boldsymbol{q}, \Omega) \zeta}
\end{aligned}
$$

Eqs. (24) and (25) involve two different dimensionless phase-mismatch functions,

$$
\begin{array}{r}
\Delta(\boldsymbol{q}, \Omega)=\left[k_{F}^{z}(\boldsymbol{q}, \Omega)+k_{F}^{z}(-\boldsymbol{q},-\Omega)-k_{S}\right] z_{0} \\
D(\boldsymbol{q}, \Omega)=\left[k_{F}^{z}(\boldsymbol{q}, \Omega)+k_{F}-k_{S}^{z}(\boldsymbol{q}, \Omega)\right] z_{0} .
\end{array}
$$


We should notice that, in the perfect phase-matched case the linearizedfluctuation analysis predicts that for large interaction lengths the fundamental field should evolve to a perfect squeezed vacuum [37]. This is in contradiction with the linearization requirement that at frequency $\omega$ the amplitude of the fluctuations should be smaller than the mean value of the of the field [38]. Comparing the predictions of the linearized analysis for traveling wave SHG with the stochastic integration of the full nonlinear propagation equations obtained in the positive $\mathrm{P}$ representation [38, 39, 40], the approximation can be considered valid for interaction lengths $\zeta \lesssim 4$ for the quantities considered here [34].

The different terms in Eqs. (24) and (25) have a clear physical interpretation: The first term in the rhs of Eq. 24 reflects the splitting inside the crystal of photons of the strong homogeneous second-harmonic wave generated by the pump into two fundamental photons with opposite frequency offsets $\Omega$ and $-\Omega$ and transverse wave vectors $\boldsymbol{q}$ and $-\boldsymbol{q}$. This process (which we call Process I) generates a coupling between the amplitudes $\hat{a}_{F}(z, \boldsymbol{q}, \Omega)$ and $\hat{a}_{F}^{\dagger}(z, \boldsymbol{q}, \Omega)$. The second term in the rhs of Eq. (24) describes the frequency down-conversion of a second-harmonic wave with $(\boldsymbol{q}, \Omega)$, into a fundamental wave with $(\boldsymbol{q}, \Omega)$ which translates into a coupling between the field operators $\hat{a}_{F}(z, \boldsymbol{q}, \Omega)$ and $\hat{a}_{S}(z, \boldsymbol{q}, \Omega)$ (Process II). Energy conservation implies that this frequency-changing process occurs under the radiation of a fundamental pump photon. The reverse process (Process III) acts as a source of second-harmonic photons and corresponds to the rhs of Eq.(25). A large phase mismatch results in fast spatial oscillations of the source term, what reduces the efficiency of a particular process. Therefore Process I will be important provided for $\Delta(\boldsymbol{q}, \Omega) \zeta \ll 1$, whereas processes II and III will be efficient for $D(\boldsymbol{q}, \Omega) \zeta \ll 1$. In the paraxial and monochromatic approximation the longitudinal wavenumber can be written as

$$
k_{i}^{z}(\boldsymbol{q}, \Omega)=k_{i}+\left[\frac{\omega_{i} n_{i}^{\prime}}{c}+\frac{k_{i}}{\omega_{i}}\right] \Omega+\frac{k_{i}^{\prime \prime}}{2} \Omega^{2}-\frac{q^{2}}{2 k_{i}},
$$

where $k_{i}^{\prime \prime}=\partial^{2} k / \partial \omega^{2}$ and $n_{i}^{\prime}=\partial n / \partial \omega$ evaluated at $\omega=\omega_{i}$. Then [46]

$$
\begin{aligned}
& \Delta(\boldsymbol{q}, \Omega)=\Delta s+\operatorname{sign}\left(k_{F}^{\prime \prime}\right) \frac{\Omega^{2}}{\Omega_{2}^{2}}-\frac{q^{2}}{q_{2}^{2}}, \\
& D(\boldsymbol{q}, \Omega)=\Delta s-\frac{\Omega}{\Omega_{1}}+\operatorname{sign}\left(k_{F}^{\prime \prime}\right) \frac{\Omega^{2}}{4 \Omega_{2}^{2}}-\frac{1}{4}\left(1-\frac{\Delta k}{2 k_{F}}\right) \frac{q^{2}}{q_{2}^{2}}
\end{aligned}
$$

where $q_{2}=\sqrt{k_{F} / z_{0}}, \Omega_{1}=\left(\frac{\omega}{c}\left(2 n_{S}^{\prime}-n_{F}^{\prime}\right) z_{0}\right)^{-1}$ and $\Omega_{2}=\left(\left|k_{F}^{\prime \prime}\right| z_{0}\right)^{-1 / 2}$.

It is convenient to introduce a vectorlike notation by defining [46]

$$
\boldsymbol{a}(z, \boldsymbol{q}, \Omega)=\left(\begin{array}{c}
\hat{a}_{F}(z, \boldsymbol{q}, \Omega) \\
\hat{a}_{S}(z, \boldsymbol{q}, \Omega)
\end{array}\right) ; \boldsymbol{a}^{\dagger}(z, \boldsymbol{q}, \Omega)=\left(\begin{array}{c}
\hat{a}_{F}^{\dagger}(z, \boldsymbol{q}, \Omega) \\
\hat{a}_{S}^{\dagger}(z, \boldsymbol{q}, \Omega)
\end{array}\right) .
$$


The solution of Eqs. (24) and (25) can be expressed in the form of a compact input-output transformation which connects the field operators at the exit plane of the crystal with those at the input plane

$$
\hat{a}_{i}(z, \boldsymbol{q}, \Omega)=\boldsymbol{U}_{i}(z, \boldsymbol{q}, \Omega) \cdot \boldsymbol{a}(0, \boldsymbol{q}, \Omega)+\boldsymbol{V}_{i}(z, \boldsymbol{q}, \Omega) \cdot \boldsymbol{a}^{\dagger}(0,-\boldsymbol{q},-\Omega) .
$$

This transformation involves 8 complex coefficients: the components the vectors $U_{F}=\left(U_{F F}, U_{F S}\right), U_{S}=\left(U_{S F}, U_{S S}\right), \boldsymbol{V}_{F}=\left(V_{F F}, V_{F S}\right)$ and $\boldsymbol{V}_{S}=\left(V_{S F}, V_{S S}\right)$. They that can be determined by solving the propagation equations.

Analytical expressions for the coefficients of the input-output transformation Eqs. (32) were obtained in [37] for $q=0$ and $\Omega=0$. In the general case, however, no analytical solution is known, so the coefficients have to be determined by numerical integration of Eqs.(24) and (25)

\subsection{Quantum image processing}

We consider an optical device represented in Fig.9: the $\chi^{(2)}$-nonlinear crystal pumped at a frequency $\omega$ enclosed in a two-lens telescopic system. The field distribution injected into the NL crystal will be the spatial Fourier transform of the original image. After processing, another lens will perform the backtransformation into real space. This two-lens imaging configuration maps any points of object and image plane onto a plane wave with a given transverse wave vector. This configuration is similar to the one based on parametric down conversion used in $[41,42]$. In terms of image processing, we consider an input image at second harmonic frequency $2 \omega$. This optical device is expected to deliver a pair of symmetric amplified versions of the input image at both fundamental and second-harmonic frequency [34].

\section{Object plane}

$$
\chi^{(2)} \text { nonlinear crystal }
$$

Image plane

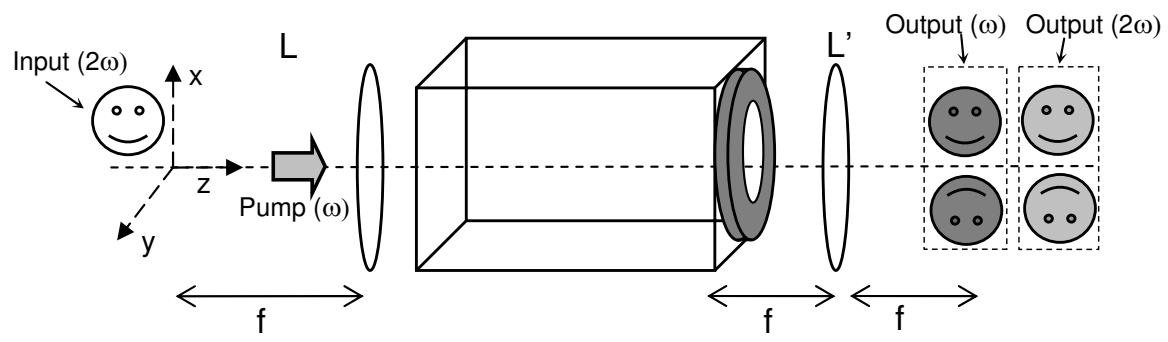

Fig. 9. Scheme of an optical device based on second harmonic generation. The nonlinear crystal, pumped at frequency $\omega$, is enclosed in a two-lens telescopic system. In the output plane of the crystal, a pupil of finite with represents the finite spatial bandwidth of the system.

We will discuss the results in terms of plane waves with given wave vectors, since the telescopic system converts these wave vectors into positions in the 
transverse plane. Furthermore, we will assume the temporal evolution of the input image to be slow and put $\Omega \rightarrow 0$ for the calculation of the output images.

The nonlinear crystal is pumped only at a frequency $\omega$. Considering a vanishing collinear phase mismatch $\Delta k=0$, Eqs. (22) and (23) lead to

$$
\begin{gathered}
\widetilde{c}_{F}(\zeta)=e^{\imath \phi_{F}^{(0)}} \operatorname{sech}(\zeta) \\
\widetilde{c}_{S}(\zeta)=e^{\imath \phi_{F}^{(0)}} \tanh (\zeta)
\end{gathered}
$$

where $\phi_{F}^{(0)}$ is the phase of the pump field.

The input signal at frequency $2 \omega$ is described by a coherent state $\left|\alpha_{i n}\right\rangle$ characterized by a complex amplitude $\alpha_{i n}(\boldsymbol{q}, \Omega)$. With respect to the frequency $\omega,\left|\alpha_{i n}\right\rangle$ is assumed to be in the vacuum state. Therefore we have

$$
\begin{aligned}
& \hat{a}_{S}(0, \boldsymbol{q}, \Omega)\left|\alpha_{i n}\right\rangle=\alpha_{i n}(\boldsymbol{q}, \Omega)\left|\alpha_{i n}\right\rangle \\
& \hat{a}_{F}(0, \boldsymbol{q}, \Omega)\left|\alpha_{i n}\right\rangle=0
\end{aligned}
$$

In parametric down-conversion according to the symmetry properties of $\alpha_{i n}(\boldsymbol{q}, \Omega)$ one distinguishes between a phase-insensitive regime, which corresponds to an input signal confined to one half of the object plane of the optical device $\left(\alpha_{i n}(\boldsymbol{q}, \Omega)=0\right.$ for $\left.q_{y}<0\right)$ [19], and the phase sensitive regime, which refers to the case of symmetrical input signal, i.e. when $\alpha_{i n}(\boldsymbol{q}, \Omega)=\alpha_{i n}(-\boldsymbol{q},-\Omega)[42]$. In the following we discuss these two regimes for the SHG system.

\section{A. Phase-insensitive configuration}

The input-output transformations given by Eqs. (32) For the input defined by Eq. (35) the input-output transformations (32) one obtains that the fundamental output field is given by

$$
\begin{gathered}
\left\langle\hat{a}_{F}^{\dagger}(\zeta, \boldsymbol{q}, \Omega) \hat{a}_{F}(\zeta, \boldsymbol{q}, \Omega)\right\rangle=(2 \pi)^{3} \delta^{(3)}(0)\left(\left|V_{F F}(\zeta, \boldsymbol{q}, \Omega)\right|^{2}+\left|V_{F S}(\zeta, \boldsymbol{q}, \Omega)\right|^{2}\right) \\
+\left|U_{F S}(\zeta, \boldsymbol{q}, \Omega)\right|^{2}\left|\alpha_{i n}(\boldsymbol{q}, \Omega)\right|^{2}+\left|V_{F S}(\zeta, \boldsymbol{q}, \Omega)\right|^{2}\left|\alpha_{i n}(-\boldsymbol{q},-\Omega)\right|^{2}
\end{gathered}
$$

which indeed is independent on the phase of the input signal. Four different contributions can be distinguished: the first two terms on the rhs of Eq. (36) are independent of the strength of the input wave and correspond to spontaneous parametric fluorescence, which takes place in the crystal even in the absence of any coherent input signal. The two other contributions are proportional to the intensity of the input wave at $(\boldsymbol{q}, \Omega)$ and $(-\boldsymbol{q},-\Omega)$, respectively. Because of the particular injection scheme considered here, for a given wave vector $\boldsymbol{q}$ at which the output is considered, only one of these two terms is nonvanishing. For an object confined to the upper part of the object plane, at the output one obtains two output images. One confined to the 
upper output plane is an amplified version of the input image and has an intensity given by $\left|U_{F S}(\zeta, \boldsymbol{q}, \Omega)\right|^{2}\left|\alpha_{i n}(\boldsymbol{q}, \Omega)\right|^{2}$. The other is confined to the lower output plane with an intensity given by $\left|V_{F S}(\zeta, \boldsymbol{q}, \Omega)\right|^{2}\left|\alpha_{i n}(-\boldsymbol{q},-\Omega)\right|^{2}$ and corresponds to a reversed amplified version of the input image. The physical underlying mechanism is Process I, second-harmonic photons generated by the strong pump wave inside the crystal decay into pairs of fundamental twin photons propagating in opposite directions [19].

The second-harmonic output intensity is given by a similar

$$
\begin{gathered}
\left\langle\hat{a}_{S}^{\dagger}(\zeta, \boldsymbol{q}, \Omega) \hat{a}_{S}(\zeta, \boldsymbol{q}, \Omega)\right\rangle=(2 \pi)^{3} \delta^{(3)}(0)\left(\left|V_{S F}(\zeta, \boldsymbol{q}, \Omega)\right|^{2}+\left|V_{S S}(\zeta, \boldsymbol{q}, \Omega)\right|^{2}\right) \\
+\left|U_{S S}(\zeta, \boldsymbol{q}, \Omega)\right|^{2}\left|\alpha_{i n}(\boldsymbol{q}, \Omega)\right|^{2}+\left|V_{S S}(\zeta, \boldsymbol{q}, \Omega)\right|^{2}\left|\alpha_{i n}(-\boldsymbol{q},-\Omega)\right|^{2}
\end{gathered}
$$

The second-harmonic output also displays both and amplified and a phaseconjugate-amplified version of the input image. Here the underlying mechanism is different, it is not the simultaneous generation of two second-harmonic waves with opposite wave vectors and frequency offsets rather than the frequency up conversion of two fundamental photons (Process III).

The efficiency of these mechanisms can be quantified defining for each of the four output images a local phase insensitive gain as the ratio of the intensity of the output wave to the intensity input wave $\left|\alpha_{i n}(\boldsymbol{q}, \Omega)\right|^{2}$. Considering a pupil of finite aperture and adequate dimensions located at the output plane of the crystal then the output intensities due to spontaneous processes can be neglected [41]. In this situation the gains are given by

$$
\begin{aligned}
& G_{F}(\zeta,+\boldsymbol{q},+\Omega)=\frac{\left\langle\hat{a}_{F}^{\dagger}(\zeta,+\boldsymbol{q},+\Omega) \hat{a}_{F}(\zeta,+\boldsymbol{q},+\Omega)\right\rangle}{\left|\alpha_{i n}(\boldsymbol{q}, \Omega)\right|^{2}}=\left|U_{F S}(\zeta, \boldsymbol{q}, \Omega)\right|^{2} \\
& G_{F}(\zeta,-\boldsymbol{q},-\Omega)=\frac{\left\langle\hat{a}_{F}^{\dagger}(\zeta,-\boldsymbol{q},-\Omega) \hat{a}_{F}(\zeta,-\boldsymbol{q},-\Omega)\right\rangle}{\left|\alpha_{i n}(\boldsymbol{q}, \Omega)\right|^{2}}=\left|V_{F S}(\zeta, \boldsymbol{q}, \Omega)\right|^{2} \\
& G_{S}(\zeta,+\boldsymbol{q},+\Omega)=\frac{\left\langle\hat{a}_{S}^{\dagger}(\zeta,+\boldsymbol{q},+\Omega) \hat{a}_{S}(\zeta,+\boldsymbol{q},+\Omega)\right\rangle}{\left|\alpha_{i n}(\boldsymbol{q}, \Omega)\right|^{2}}=\left|U_{S S}(\zeta, \boldsymbol{q}, \Omega)\right|^{2} \\
& G_{S}(\zeta,-\boldsymbol{q},-\Omega)=\frac{\left\langle\hat{a}_{S}^{\dagger}(\zeta,-\boldsymbol{q},-\Omega) \hat{a}_{S}(\zeta,-\boldsymbol{q},-\Omega)\right\rangle}{\left|\alpha_{i n}(\boldsymbol{q}, \Omega)\right|^{2}}=\left|V_{S S}(\zeta, \boldsymbol{q}, \Omega)\right|^{2}
\end{aligned}
$$

Fig. 10 shows the phase-insensitive gains as functions of the interaction length inside the crystal. At the input plane we have $G_{F}(\zeta=0,+\boldsymbol{q},+\Omega)=$ $G_{F}(\zeta=0,-\boldsymbol{q},-\Omega)=G_{S}(\zeta=0,-\boldsymbol{q},-\Omega)=0$ and $G_{S}(\zeta=0,+\boldsymbol{q},+\Omega)=1$, which simply identifies the chosen input. Increasing the interaction length, $G_{S}(\zeta,+\boldsymbol{q},+\Omega)$ decreases and $G_{F}(\zeta,+\boldsymbol{q},+\Omega)$ increases while $G_{F}(\zeta,-\boldsymbol{q},-\Omega)$ and $G_{S}(\zeta,-\boldsymbol{q},-\Omega)$ remain very small. At small interaction length Process II is dominant. At $\zeta \simeq 1.4$, the injected second-harmonic signal is fully downconverted. Further increase of the interaction length leads to a second step in the signal processing, which, for small wave numbers (solid lines in Fig. 10), is characterized by a rapid and symmetric growth of both the fundamental gains at $+\boldsymbol{q}$ and $-\boldsymbol{q}$. This is the manifestation of the photon down-conversion Process I. This region of the crystal acts mainly as an OPA with a $z$-dependent 
pump. However, the presence of a weak residual pump field at frequency $\omega$ allows to a partial frequency up-conversion of the amplified waves at fundamental frequency through Process III. This mechanism is responsible of a slow increase of the gains at $+\boldsymbol{q}$ and $-\boldsymbol{q}$ when increasing interaction length, and leads to the formation of the two phase-conjugate output images at the second-harmonic frequency.
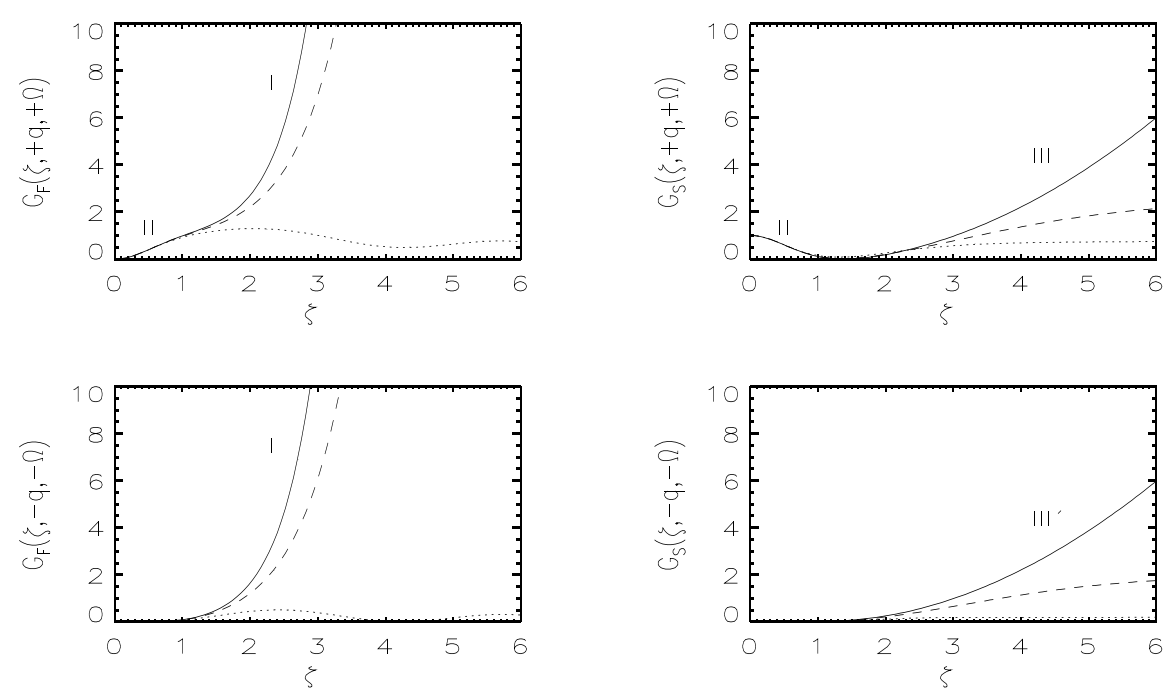

Fig. 10. Phase-insensitive gains as a function of the interaction length $\zeta$ for waves with $\Omega=0$ and different wave numbers: $q=0.5$ (solid line), $q=1.2$ (dashed line), and $q=1.6$ (dotted line) (in units of $q_{2}=\sqrt{k_{F} / z_{0}}$. The symbols I, II and III refer to the corresponding dominant elementary process. Process III' is the same as process III but with $\boldsymbol{q}$ replaced by $\boldsymbol{- q}$

Due to diffraction, which is encoded in the dependence of the phase mismatch functions $D(\boldsymbol{q}, \Omega)$ and $\Delta(\boldsymbol{q}, \Omega)$ on $\boldsymbol{q}$ and $\Omega$, the gains at high transverse wave number will be reduced and for $q>\sqrt{2}$ the input signal is no longer amplified; it rather has a oscillatory behavior as function of the interaction length [34, 43]. Therefore only a finite disk-shaped portion of the input image centered on the beam axis will be efficiently processed [34] similarly to what happens in a perfect-matched OPA [19].

\section{B. Phase-sensitive configuration}

We consider here a symmetrical input image $\alpha_{i n}(-\boldsymbol{q},-\Omega)=\alpha_{i n}(\boldsymbol{q}, \Omega)$. For static signals, this translates into a symmetry of the input image with respect to the beam axis. In the case of the OPA, it is well known for this symmetric images, the output is the result of the coherent superposition of 
both twin waves produced in an elementary down-conversion process [44]. The amplification is, therefore, phase sensitive, which is one of the requisites for amplifying an image without deteriorating its signal-to-noise ratio $[24,42,45]$.

When the image inserted in the SHG system is symmetric with respect to the beam axis the output images at each frequency will display the same symmetry. Under the same assumptions than for the phase-insensitive case, the ratio of the intensity in a given portion of each output image to the one in the corresponding part of the input image defines the gains

$$
\begin{gathered}
G_{F}^{\left(\phi_{i n}\right)}(\zeta, \boldsymbol{q}, \Omega)=\left|U_{F S}(\zeta, \boldsymbol{q}, \Omega) e^{\imath \phi_{i n}}+V_{F S}(\zeta, \boldsymbol{q}, \Omega) e^{-\imath \phi_{i n}}\right|^{2}, \\
G_{S}^{\left(\phi_{i n}\right)}(\zeta, \boldsymbol{q}, \Omega)=\left|U_{S S}(\zeta, \boldsymbol{q}, \Omega) e^{\imath \phi_{i n}}+V_{S S}(\zeta, \boldsymbol{q}, \Omega) e^{-\imath \phi_{i n}}\right|^{2},
\end{gathered}
$$

which both depend on the phase of the input signal $\phi_{i n}$. For simplicity, we only consider input images with an homogeneous phase $\alpha_{i n}(\boldsymbol{q}, \Omega)=$ $\left|\alpha_{i n}(\boldsymbol{q}, \Omega)\right| e^{\imath \phi_{i n}}$.

Fig. 11 shows the phase dependence of the gains for different transverse wave numbers (which in the telescopic system correspond to different regions of the transverse plane). On the optical axis $(\boldsymbol{q}=0)$, both gains reach maximum values for $\phi_{i n}=\pi / 2+n \pi$ and minumum at $\phi_{i n}=n \pi$ [34]. For off-axis regions of the transverse plane, one observes a shift in the position of the maximum and minimum gains (dashed and dotted lines in Fig. 11). This implies that for an input image with an homogeneous phase, the maximal gain condition can only be satisfied at one point of the transverse plane. However, it should be possible to compensate this through a displacement of the nonlinear crystal with respect to the lenses, which amounts to superposing a parabolic phase profile to the overall phase of the input image, as was considered for the OPA in Ref. [42].
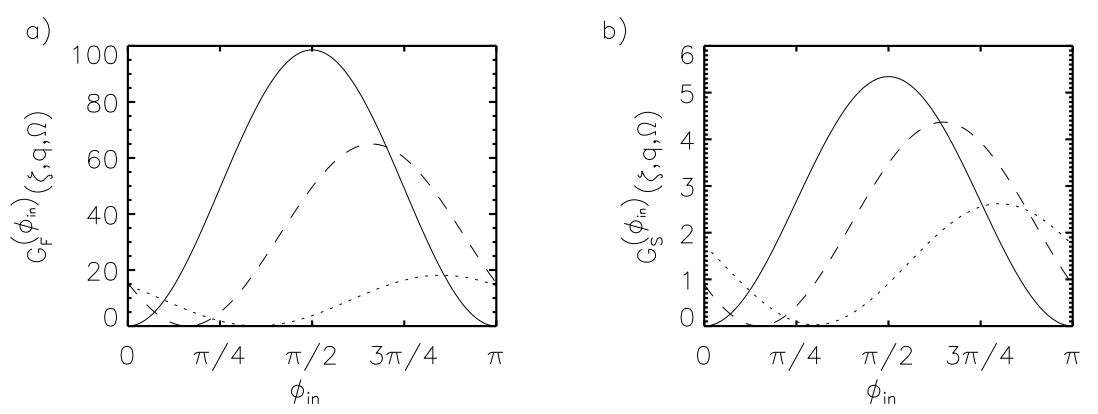

Fig. 11. Phase-sensitive gains for fundamental (a) and second harmonic (b) fields as a function of the phase of the input signal (in radians) for different values of the transverse wave number $q=0$ (solid line) $q=1$ (dashed line) and $q=1.4$ (dotted line). Interaction length $\zeta=3.32$ 
The effect of diffraction is similar to the phase-insensitive case, the image processing will be efficient within a region of finite with centered on the beam axis, whereas outside this region, the nonlinear crystal will behave as a transparent medium [34].

Now we address the noise properties of the system we are considering, which are determined by the quantum fluctuations of the output fields. We first define for the fundamental and second-harmonic fields the following quadrature operators at phase $\phi_{L O}$

$$
x_{i}^{\phi_{L O}}(\zeta, \boldsymbol{q}, \Omega)=\frac{1}{2}\left[a_{i}(\zeta, \boldsymbol{q}, \Omega) e^{-\imath \phi_{L O}}+a_{i}^{\dagger}(\zeta,-\boldsymbol{q},-\Omega) e^{\imath \phi_{L O}}\right]
$$

which involves the field amplitude operator $a_{i}(\zeta, \boldsymbol{q}, \Omega)$ which is related to the propagation-corrected amplitude $\hat{a}_{i}(\zeta, \boldsymbol{q}, \Omega)$ as follows

$$
a_{i}(\zeta, \boldsymbol{q}, \Omega)=\exp \left\{\imath\left[k_{i}^{z}(\boldsymbol{q}, \Omega)-k_{i}\right] z_{0} \zeta\right\} \hat{a}_{i}(\zeta, \boldsymbol{q}, \Omega) .
$$

The exponential factor simply restores the phase accumulated during propagation, which had been factorized out in Eq. (15) for technical reasons. Unlike in the quantities considered before, now this phase factor is important as illustrated in [25].

The variance of the quadrature operator $x_{i}^{\left(\phi_{L O}\right)}(\zeta, \boldsymbol{q}, \Omega)$ defines the spectrum of squeezing $S_{i}^{\left(\phi_{L O}\right)}(\zeta, \boldsymbol{q}, \Omega)$ through the relation

$$
\left\langle x_{i}^{\left(\phi_{L O}\right)}(\zeta, \boldsymbol{q}, \Omega), x_{i}^{\left(\phi_{L O}\right)}\left(\zeta, \boldsymbol{q}^{\prime}, \Omega^{\prime}\right)\right\rangle=\frac{1}{4} \delta^{(2)}\left(\boldsymbol{q}+\boldsymbol{q}^{\prime}\right) \delta\left(\Omega+\Omega^{\prime}\right) S_{i}^{\left(\phi_{L O}\right)}(\zeta, \boldsymbol{q}, \Omega)
$$

with the usual definition of the variance $\langle f, g\rangle=\langle f g\rangle-\langle f\rangle\langle g\rangle$. In the case of photodetectors with perfect quantum efficiency, the spectrum of the squeezing coincides with the spectral density for photocurrent fluctuations, normalized to the shot noise level, as measured in a homodyne detection scheme. $\phi_{L O}$ represents the phase of the local oscillator used in this detection setup.

Fig. 12 shows $S_{\sigma}^{\left(\phi_{L O}\right)}(\zeta, \boldsymbol{q}, \Omega)$ as a function of the local oscillator phase. As it happens for a one-mode squeezing transformation, changing the local oscillator phase allows one to explore the shape of the uncertainty region covered by the quantum fluctuations of the field. The maximum of $S_{\sigma}^{\left(\phi_{L O}\right)}(\zeta, \boldsymbol{q}, \Omega)$ corresponds to the local oscillator pointing along the quadrature with stretched fluctuations, whereas the minimum indicates the direction of the squeezed quadrature. The effects of diffraction can be analyzed considering different values of $\boldsymbol{q}$. It is clear that the maximum and minimum values of the spectrum of squeezing are shifted for different values of $\boldsymbol{q}$. This shift can be interpreted as a rotation of the axis of the uncertainty region, as in the OPA [25]. Simultaneously, a reduction of the amplitude of the oscillations of the spectrum of the squeezing indicates a reduction of the squeezing effect with larger $\boldsymbol{q}$, with the consequence that the uncertainty region recovers more and more the circular shape characteristic for a coherent state. 

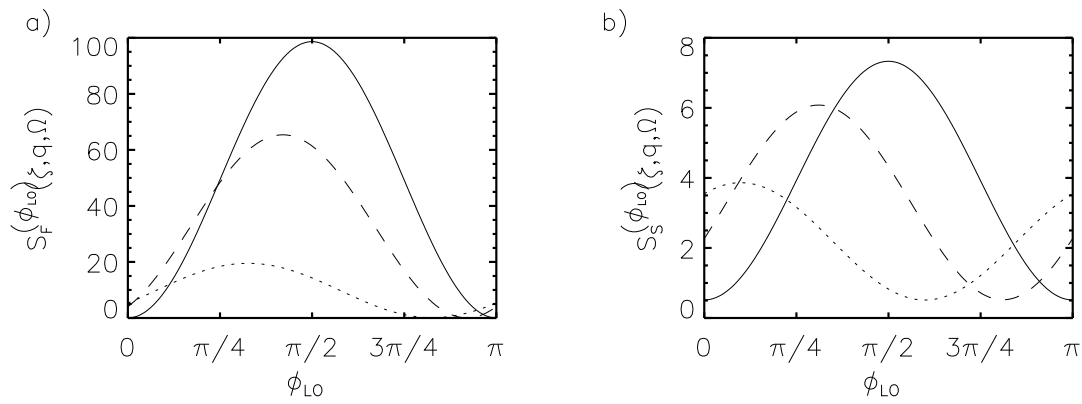

Fig. 12. Spectrum of squeezing $S_{\sigma}^{\left(\phi_{L O}\right)}(\zeta, \boldsymbol{q}, \Omega)$ for fundamental (a) and second harmonic (b) fields as a function of the phase of the local oscillator (in radians) for $\Omega=0$ and different values of the transverse wave vector number $q=0$ (solid line), $q=1$ (dashed line) and $q=1.4$ (dotted line). Interaction length $\zeta=3.32$

To appreciate the performances of the SHG device with respect to the noiseless signal processing, it is convenient to consider the same detection scheme than considered for noiseless amplification in OPA [19]. It consist in measuring the sum of the photocurrents from two symmetric pixels in the output plane. For such device, the noise figure is given by the ratio of the intensity-squeezing spectrum to the phase-sensitive gain [34]

$$
F_{i}(q)=\frac{S_{i}^{\phi_{\sigma}^{\text {out }}}(\zeta, \boldsymbol{q}, \Omega)}{G_{i}^{\phi_{i n}}(\zeta, \boldsymbol{q}, \Omega)},
$$

where

$$
\begin{gathered}
\phi_{F}^{\text {out }}=\arg \left[U_{F S}(\zeta, \boldsymbol{q}, \Omega) e^{\imath \phi i n}+V_{F S}(\zeta, \boldsymbol{q}, \Omega) e^{-\imath \phi i n}+\left[k_{F}^{z}(\boldsymbol{q}, \Omega)-k_{F}\right] z_{0} \zeta(, 48)\right. \\
\phi_{S}^{\text {out }}=\arg \left[U_{S S}(\zeta, \boldsymbol{q}, \Omega) e^{\imath \phi i n}+V_{S S}(\zeta, \boldsymbol{q}, \Omega) e^{-\imath \phi i n}+\left[k_{S}^{z}(\boldsymbol{q}, \Omega)-k_{S}\right] z_{0} \zeta(49)\right.
\end{gathered}
$$

Fig. 13 shows the noise figure for the fundamental and second harmonic outputs choosing the phase of the input signal such that the phase-sensitive gains are maximal at $q=0$. Since we are considering linearized propagation equations for the field operators the noise figure can be never less than 1 (dotted line) [45] which corresponds to a noiseless operation.

In the domain of wave numbers for which image processing is efficient we find that the fundamental output shows the same level of noise as the input image $\left(F_{S} \approx 1\right)$ so the SHG device operates without adding noise to the signal. For the second-harmonic field the noise figure is slightly above 1, implying a a degradation of the signal to noise ratio in the output secondharmonic image. However, increasing the interaction length, the figure noise is seen to approach 1 for the spatial frequencies inside the bandwidth for image processing. Finally one notes that, for large transverse wave number the noise figure for the second-harmonic frequency is equal to 1 , since the 

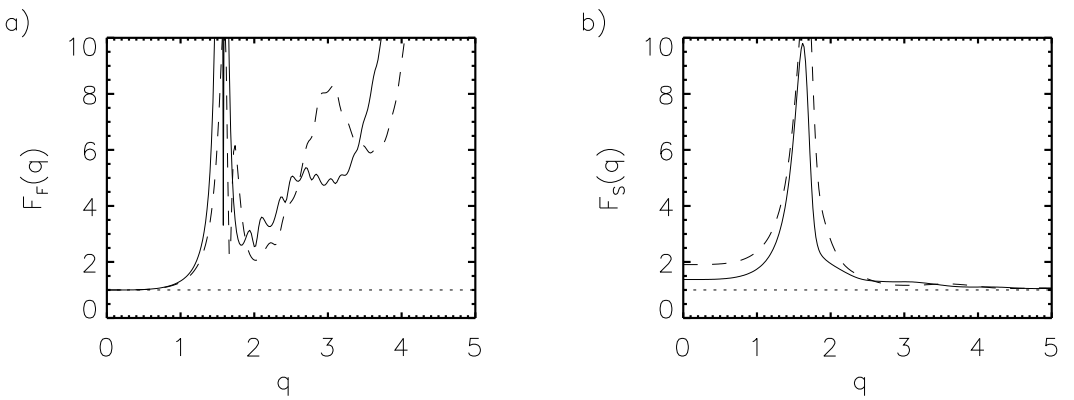

Fig. 13. Noise figure for fundamental (a) and second harmonic (b) fields as a function of the transverse wave number for two different values of the interaction length $\zeta=3.32$ (solid line) and $\zeta=2.5$ (dashed line). As a dotted line, the smallest possible value $F_{i}(q)=1$ for a linear system

input signal is unaffected by the system in this region, whereas for the noise figure with respect to the fundamental frequency diverges, as a consequence of a vanishing output intensity at $\omega$ in the limit $|\boldsymbol{q}| \rightarrow \infty$.

\section{Quantum image processing in type-II second-harmonic generation}

In this section we will explore the possibilities offered by type II second harmonic generation for image processing, where the polarization degree of freedom at the fundamental frequency plays a very important role, as discussed in Sec. 2 at the classical level. Here we will consider the role of quantum fluctuations in the telescopic traveling wave configuration shown in Figure 14 which is similar to the one considered in Sec. 3. However while in that case the image was introduced as a second harmonic signal, here, since we have two orthogonally polarized fundamental fields we introduce the image on the fundamental fields, as in Sec. 2. We will show that with traveling-wave type II SHG it is possible to noiseless up-convert the part of an image with a given polarization while the part with orthogonal polarization is noiseless amplified. In the following sections we will first present the propagation equations for this system, as an extension of the equations presented in subsection 3.2. Then we will discuss the imaging properties of this system in two different configurations.

\subsection{Propagation equations}

Following the same steps than in subsection 3.2 the propagation equations for the propagation-corrected Fourier amplitudes are 
Object plane

$\chi^{(2)}$ nonlinear crystal

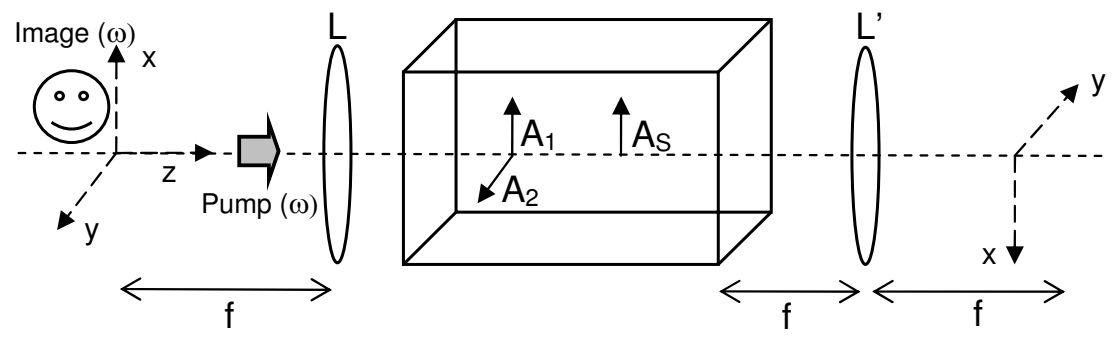

Fig. 14. Scheme of an optical device based on type II second harmonic generation. The nonlinear crystal, pumped at frequency $\omega$, is enclosed in a two-lens telescopic system.

$$
\begin{array}{r}
\frac{\partial}{\partial z} \hat{A}_{S}(z, \boldsymbol{q}, \Omega)=+K \int d^{2} q^{\prime} d \Omega^{\prime} \hat{A}_{1}\left(z, \boldsymbol{q}^{\prime}, \Omega^{\prime}\right) \hat{A}_{2}\left(z, \boldsymbol{q}-\boldsymbol{q}^{\prime}, \Omega-\Omega^{\prime}\right) \\
\times \exp \left\{l\left[k_{1}^{z}\left(\boldsymbol{q}^{\prime}, \Omega^{\prime}\right)+k_{2}^{z}\left(\boldsymbol{q}-\boldsymbol{q}^{\prime}, \Omega-\Omega^{\prime}\right)-k_{S}^{z}(\boldsymbol{q}, \Omega)\right] z\right\} \\
\frac{\partial}{\partial z} \hat{A}_{1}(z, \boldsymbol{q}, \Omega)=-K \int d^{2} q^{\prime} d \Omega^{\prime} \hat{A}_{2}^{\dagger}\left(z, \boldsymbol{q}^{\prime}, \Omega^{\prime}\right) \hat{A}_{S}\left(z, \boldsymbol{q}+\boldsymbol{q}^{\prime}, \Omega+\Omega^{\prime}\right) \\
\times \exp \left\{l\left[k_{S}^{z}\left(\boldsymbol{q}+\boldsymbol{q}^{\prime}, \Omega+\Omega^{\prime}\right)-k_{1}^{z}(\boldsymbol{q}, \Omega)-k_{2}^{z}\left(\boldsymbol{q}^{\prime}, \Omega^{\prime}\right)\right] z\right\} \\
\frac{\partial}{\partial z} \hat{A}_{2}(z, \boldsymbol{q}, \Omega)=-K \int d^{2} q^{\prime} d \Omega^{\prime} \hat{A}_{1}^{\dagger}\left(z, \boldsymbol{q}^{\prime}, \Omega^{\prime}\right) \hat{A}_{S}\left(z, \boldsymbol{q}+\boldsymbol{q}^{\prime}, \Omega+\Omega^{\prime}\right) \\
\times \exp \left\{\imath\left(k_{S}^{z}\left(\boldsymbol{q}+\boldsymbol{q}^{\prime}, \Omega+\Omega^{\prime}\right)-k_{2}^{z}(\boldsymbol{q}, \Omega)-k_{1}^{z}\left(\boldsymbol{q}^{\prime}, \Omega^{\prime}\right)\right] z\right\}
\end{array}
$$

where the index 1,2 and $S$ correspond to de $x$-polarized fundamental field, the $y$-polarized fundamental field and the second harmonic field respectively. This equations have a simple physical interpretation: the generation of a given field mode is seen as the result of all possible three wave processes that fulfill energy and transverse momentum conservation.

Now we use a linearization approach as in the previous section. Assuming that the pump field is a strong classical field, which, inside the crystal will eventually produce an strong classical field at second harmonic frequency we have

$$
\left.\hat{A}_{i}(z, \boldsymbol{q}, \Omega)=\tilde{c}_{i}(z) \delta^{(2)}(\boldsymbol{q}) \delta(\Omega)\right)+\hat{a}_{i}(z, \boldsymbol{q}, \Omega),
$$

where $i$ stands for $1,2, \mathrm{~S}$ and $\tilde{c}_{i}(z)$ are the amplitudes of the classical strong monochromatic fields and $\hat{a}_{i}(z, \boldsymbol{q}, \Omega)$ are the quantum field operators associated to each field. The total power $W=\left|\tilde{c}_{1}(z)\right|^{2}+\left|\tilde{c}_{2}(z)\right|^{2}+2\left|\tilde{c}_{S}(z)\right|^{2}$ is conserved as a consequence of the conservation of the energy flux in the lossless crystal. Introducing the dimensionless characteristic interaction length $z_{0}=1 / \sqrt{2 W} K$, scaling the space as $\zeta=z / z_{0}$ and the field amplitudes as $c_{1}(z)=\tilde{c}_{1}(z) / \sqrt{W}, c_{2}(z)=\tilde{c}_{2}(z) / \sqrt{W}$ and $c_{S}(z)=\tilde{c}_{S}(z) / \sqrt{W / 2}$ and substituting Eqs. (53) in Eqs. (50) - (52) we obtain at zero order the classical equations of nonlinear optics 


$$
\begin{aligned}
\frac{d}{d \zeta} c_{1}(\zeta) & =-c_{2}^{*}(\zeta) c_{S}(\zeta) e^{-\imath \Delta s \zeta} \\
\frac{d}{d \zeta} c_{2}(\zeta) & =-c_{1}^{*}(\zeta) c_{S}(\zeta) e^{-\imath \Delta s \zeta} \\
\frac{d}{d \zeta} c_{S}(\zeta) & =+2 c_{1}(\zeta) c_{2}(\zeta) e^{\imath \Delta \zeta}
\end{aligned}
$$

where $\Delta s=\Delta k z_{0}$.

At first order one obtains the propagation equations for the quantum operators associated to the fundamental and second-harmonic fields

$$
\begin{aligned}
& \frac{\partial}{\partial \zeta} \hat{a}_{1}(\zeta, \boldsymbol{q}, \Omega)=-c_{S}(\zeta) e^{-\imath \Delta_{1}(\boldsymbol{q}, \Omega) z} \hat{a}_{2}^{\dagger}(\zeta,-\boldsymbol{q},-\Omega) \\
& -\sqrt{2} c_{2}^{*}(\zeta) e^{-\imath D_{1}(\boldsymbol{q}, \Omega) z} \hat{a}_{S}(\zeta, \boldsymbol{q}, \Omega) \\
& \frac{\partial}{\partial \zeta} \hat{a}_{2}(\zeta, \boldsymbol{q}, \Omega)=-c_{S}(\zeta) e^{\imath \Delta_{1}(-\boldsymbol{q},-\Omega) z} \hat{a}_{1}^{\dagger}(\zeta,-\boldsymbol{q},-\Omega) \\
& -\sqrt{2} c_{1}^{*}(\zeta) e^{-\imath D_{2}(\boldsymbol{q}, \Omega) z} \hat{a}_{S}(\zeta, \boldsymbol{q}, \Omega) \\
& \frac{\partial}{\partial \zeta} \hat{a}_{S}(\zeta, \boldsymbol{q}, \Omega)=+\sqrt{2} c_{2}(\zeta) e^{\imath D_{1}(\boldsymbol{q}, \Omega) z} \hat{a}_{1}(\zeta, \boldsymbol{q}, \Omega) \\
& +\sqrt{2} c_{1}(\zeta) e^{\imath D_{2}(\boldsymbol{q}, \Omega) z} \hat{a}_{2}(\zeta, \boldsymbol{q}, \Omega)
\end{aligned}
$$

the phase factors involved in the equations are

$$
\begin{aligned}
& \Delta_{1}(\boldsymbol{q}, \Omega)=z_{0}\left(k_{1}^{z}(\boldsymbol{q}, \Omega)+k_{2}^{z}(-\boldsymbol{q},-\Omega)-k_{S}\right) \\
& D_{1}(\boldsymbol{q}, \Omega)=z_{0}\left(k_{1}^{z}(\boldsymbol{q}, \Omega)+k_{2}-k_{S}^{z}(\boldsymbol{q}, \Omega)\right) \\
& D_{2}(\boldsymbol{q}, \Omega)=z_{0}\left(k_{1}+k_{2}^{z}(\boldsymbol{q}, \Omega)-k_{S}^{z}(\boldsymbol{q}, \Omega)\right)
\end{aligned}
$$

Using the vector-like notation [46]

$$
\boldsymbol{a}(z, \boldsymbol{q}, \Omega)=\left(\begin{array}{c}
\hat{a}_{1}(z, \boldsymbol{q}, \Omega) \\
\hat{a}_{2}(z, \boldsymbol{q}, \Omega) \\
\hat{a}_{S}(z, \boldsymbol{q}, \Omega)
\end{array}\right) ; \boldsymbol{a}^{\dagger}(z, \boldsymbol{q}, \Omega)=\left(\begin{array}{c}
\hat{a}_{1}^{\dagger}(z, \boldsymbol{q}, \Omega) \\
\hat{a}_{2}^{\dagger}(z, \boldsymbol{q}, \Omega) \\
\hat{a}_{S}^{\dagger}(z, \boldsymbol{q}, \Omega)
\end{array}\right),
$$

the solution of the propagation equations (57)-(59) can be written as

$$
\hat{a}_{i}(z, \boldsymbol{q}, \Omega)=\boldsymbol{U}_{i}(z, \boldsymbol{q}, \Omega) \cdot \boldsymbol{a}(0, \boldsymbol{q}, \Omega)+\boldsymbol{V}_{i}(z, \boldsymbol{q}, \Omega) \cdot \boldsymbol{a}^{\dagger}(0,-\boldsymbol{q},-\Omega)
$$

which involves 18 complex coefficients that can be determined by solving the propagation equations.

Superposed to the homogeneous pump field, an optical image will be injected into the nonlinear crystal as a coherent state $\left|\alpha_{i n}\right\rangle$. This quantum state can be written as

$$
\boldsymbol{a}(0, \boldsymbol{q}, \Omega)\left|\alpha_{i n}\right\rangle=\boldsymbol{\alpha}(q) \delta(\Omega)\left|\alpha_{i n}\right\rangle
$$


The amplitude $\boldsymbol{\alpha}=\left(\alpha_{1}(q), 0,0\right)$ encodes, within the vectorial notation defined trough Eqs. (63), the spatial distribution of the input image at the fundamental frequency with polarization $x$ and $y$, and at the second harmonic frequency, respectively. We consider here an image stationary in time and inserted in the $x$ polarization.

The quantities of interest are the numbers of photons of each field detected by a photodetector located at a given position $\rho$ of the image plane, which in the telescopic configuration considered is identified with a given transverse number. Assuming a detector whose size $\sigma_{d}$ is much smaller that the typical variation scales of the fields and with a perfect quantum efficiency, the average number of photons is given by:

$$
<\hat{N}_{i}(\zeta, q)>=\sigma_{d}<\hat{a}_{i}^{\dagger}(\zeta, q, 0) \hat{a}_{i}(\zeta, q, 0)>=\sigma_{d}\left|\alpha^{\text {out }}(q)\right|^{2}
$$

where

$$
\alpha_{i}^{\text {out }}(q)=\boldsymbol{U}_{i}(q, 0) \cdot \boldsymbol{\alpha}(q)+\boldsymbol{V}_{i}(q, 0) \cdot \boldsymbol{\alpha}^{*}(-q),
$$

represents the amplitude of the outgoing wave for the field $i=1,2, S$. The photon numbers depend on the amplitude of the input image at the wave number $q$ but also, essentially as a consequence of the photon down conversion process which takes place in the crystal, on the input wave at $-q$. The photon number variance is given by

$$
\left\langle\Delta^{2} \hat{N}_{i}(L, q)\right\rangle=\sigma_{d}\left|\alpha_{i}^{\text {out }}(q)\right|^{2}\left(1+2 \boldsymbol{V}_{i}^{*}(q, 0) \cdot \boldsymbol{V}_{i}(q, 0)\right) .
$$

The noise level of the detected image is quantified by the signal-to-noise ratio:

$$
\mathrm{SNR}_{i}=\frac{\left\langle\hat{N}_{i}(L, q)\right\rangle^{2}}{\left\langle\Delta^{2} \hat{N}_{i}(L, q)\right\rangle},
$$

which has to be compared to the signal-to-noise ratio in the input-image $\mathrm{SNR}_{1}^{i n}=\sigma_{d}\left|\alpha_{1}(q)\right|^{2}$. The noise figure is the ratio $\mathrm{SNR}_{1}^{i n} / \mathrm{SNR}_{i}$ :

$$
F_{i}=\frac{1+2 \boldsymbol{V}_{i}^{*}(q, 0) \cdot \boldsymbol{V}_{i}(q, 0)}{\left|\alpha_{i}^{\text {out }}(q)\right|^{2} /\left|\alpha_{1}(q)\right|^{2}}
$$

As stated in subsection 3.2, if the noise figure is equal to 1 the image processing is said to be noiseless, and at the level of quantum fluctuations, the quality of the image is preserved. This is the best possible situation in a system like the one we are considering here, but generally the noise figure is larger than 1, therefore a degradation of the image quality occurs which can even lead to a complete loss of information in the case of weak inputs.

\subsection{Linearly $y$-polarized pump: the frequency addition regime}

The first case we will consider is the simplest one: the pump field is taken as linearly polarized along the $y$-direction. This is expressed in the initial condition 


$$
c_{1}(0)=0 ; c_{2}(0)=1 ; c_{3}(0)=0
$$

Since no field is pumped in the orthogonal $x$-polarization, SHG cannot take place and the classical equations of nonlinear optics [27, 28, 29, 30, 31, 34] predicts that

$$
c_{1}(\zeta)=0 ; c_{2}(\zeta)=1 ; c_{3}(\zeta)=0 .
$$

The linearized propagation equations (Eqs. (57)-(59)) for the quantum operators can be easily be solved analytically. One finally obtains the following input output transformation:

$$
\begin{aligned}
\hat{a}_{1}(\zeta, q, \Omega)= & \left(\cos \left(\widetilde{D}_{1} \zeta\right)+\imath \frac{D_{1}}{2 \widetilde{D}_{1}} \sin \left(\widetilde{D}_{1} \zeta\right)\right) e^{-\imath D_{1} \zeta / 2} \hat{a}_{1}(0, q, \Omega) \\
& -\frac{\sqrt{2}}{\widetilde{D}_{1}} \sin \left(\widetilde{D}_{1} \zeta\right) e^{-\imath D_{1} \zeta / 2} \hat{a}_{S}(0, q, \Omega) \\
\hat{a}_{S}(\zeta, q, \Omega)= & \left(\cos \left(\widetilde{D}_{1} \zeta\right)-\imath \frac{D_{1}}{2 \widetilde{D}_{1}} \sin \left(\widetilde{D}_{1} \zeta\right)\right) e^{\imath D_{1} \zeta / 2} \hat{a}_{S}(0, q, \Omega) \\
& +\frac{\sqrt{2}}{\widetilde{D}_{1}} \sin \left(\widetilde{D}_{1} \zeta\right) e^{\imath D_{1} \zeta / 2} \hat{a}_{1}(0, q, \Omega) \\
\hat{a}_{2}(\zeta, q, \Omega)= & \hat{a}_{2}(0, q, \Omega)
\end{aligned}
$$

where $\widetilde{D}_{1}(q, \Omega)=\sqrt{2+D_{1}^{2}(q, \Omega) / 4}$. While the $y$-polarized image would not be affected by the propagation through the nonlinear crystal, this device is able to up-convert an $x$-polarized input image at frequency $\omega$ to frequency $2 \omega$. This comes form the dependence of the output operator $a_{s}(\zeta, q, \Omega$ ) (as well as $\left.a_{1}(\zeta, q, \Omega)\right)$ on both input operators $a_{1}(0, q, \Omega)$ and $a_{S}(0, q, \Omega)$. Since no second harmonic strong wave is created in the crystal, no photon down conversion is possible, and hence no coupling waves associated with frequencies $(q, \Omega)$ and $(-q,-\Omega)$ occurs during propagation. Comparing to the general form of the input-output transformation (64), all the coefficients $\boldsymbol{V}_{i}(q, \Omega)$ vanish in the case under consideration, and as a consequence the output does not depend on the phase of the input signal.

The gain of the upconversion process is

$$
G_{S}(\zeta, q) \equiv \frac{\left\langle\hat{N}_{S}(\zeta, q)\right\rangle}{\left\langle\hat{N}_{1}(0, q)\right\rangle}=2 \frac{\sin ^{2}\left(\tilde{D}_{1}(q, \Omega) z\right)}{\tilde{D}_{1}(q, \Omega)^{2}} .
$$

Perfect up-conversion $G_{S}=1$ is achieved if: $D_{1}(q, \Omega)=0$ and $\sin \left(\tilde{D}_{1}(q, \Omega) z\right)=$ 1. The first condition, corresponding to perfect wave vector matching defines an ensemble of points in the spatio-temporal frequency plane $(q, \Omega)$, and, in the case of static images, at most two values of $q$. The second condition, establishes that even in that case the maximum efficiency occurs only at given propagation lengths $\zeta_{k}=(\pi / \sqrt{2})(k+1 / 2)$, with $k=0,1, \ldots$

The noise figure coincides with the inverse of the up-conversion rate. 


$$
F(q)=\frac{1}{R_{u p}(q)}
$$

The input output transformation (73)-(75) is completely equivalent to the transformation performed by a beam splitter. In the case of a coherent image, and assuming that the second-harmonic input state is the vacuum, an incomplete up-conversion means that the mean intensity of the output is reduced with respect to the input image, but the amount of noise is conserved, corresponding to the one of a coherent state. As a result, a deterioration of the image takes place and the noise figure for the transmitted image is equal to the inverse of the transmission coefficient of the beam splitter. The quantum noise present in the up-converted image can be interpreted as the superposition of the noise already present in the input image, which is partially transmitted together with the image itself, and the noise originally present in the second harmonic modes, partially reflected by the beam splitter. Therefore a reduction of the noise level in the output image can only be achieved by reducing the fluctuations present in the second harmonic input field. This can be done by injecting a squeezed vacuum at the second harmonic frequency instead of normal vacuum. By properly choosing the squeezed quadrature, this contribution to the total noise level in the image can be reduced, and one may approach, in the limit of a perfect squeezed quadrature, a noise figure of 1, i.e. a noiseless image processing. In the next section we will consider an slightly different setup which allows to achieve a noiseless up-conversion without any additional source of nonclassical light.

\section{3 $45^{\circ}$ Linearly polarized pump: noiseless up-conversion and amplification}

We consider the case where the pump field is linearly polarized at $45^{\circ}$ with respect to the axis $x$. The initial condition for the classical strong fields is

$$
c_{1}(0)=c_{2}(0)=1 / \sqrt{2} ; c_{s}(0)=0
$$

Since now the fundamental field is pumped at the two orthogonal polarizations, a strong second harmonic field will be generated inside the crystal. Considering perfect matched wave vector number, the solution of the classical equations $[27,28,29,30,31,34]$ is particulary simple and reads [47]:

$$
c_{1}(\zeta)=c_{2}(\zeta)=\operatorname{sech}(\zeta) / \sqrt{2} ; c_{s}(\zeta)=\tanh (\zeta)
$$

It is useful to introduce a polarization basis rotated by an angle of $45^{\circ}$ with respect to the basis $(x, y)[36,37,46]$ :

$$
\hat{a}_{ \pm}(\zeta, q)=\frac{1}{\sqrt{2}}\left(\hat{a}_{1}(\zeta, q) \pm \hat{a}_{2}(\zeta, q)\right)
$$

In this basis $c_{+}(\zeta)=\operatorname{sech}(\zeta)$ and $c_{-}(\zeta)=0$. The propagation equations in this new basis can easily be derived from Eqs.(57)-(59) and one finds 


$$
\begin{aligned}
\frac{\partial}{\partial \zeta} & \hat{a}_{ \pm}+(\zeta, \boldsymbol{q}, \Omega)=-c_{S}(\zeta) g_{ \pm}(\zeta, \boldsymbol{q}, \Omega) \hat{a}_{+}^{\dagger}(\zeta,-\boldsymbol{q},-\Omega) \\
& +c_{S}(\zeta) g_{\mp}(\zeta, \boldsymbol{q}, \Omega) \hat{a}_{-}^{\dagger}(\zeta,-\boldsymbol{q},-\Omega)-\sqrt{2} c_{+}^{*}(\zeta) h_{ \pm}(\zeta, \boldsymbol{q}, \Omega) \hat{a}_{S}(\zeta, \boldsymbol{q}, \Omega)(81) \\
\frac{\partial}{\partial \zeta} & \hat{a}_{S}(\zeta, \boldsymbol{q}, \Omega)=\sqrt{2} c_{+}^{*}(\zeta) h_{+}^{*}(\zeta, \boldsymbol{q}, \Omega) \hat{a}_{+}(\zeta, \boldsymbol{q}, \Omega) \\
& +\sqrt{2} c_{+}^{*}(\zeta) h_{-}^{*}(\zeta, \boldsymbol{q}, \Omega) \hat{a}_{-}(\zeta, \boldsymbol{q}, \Omega)
\end{aligned}
$$

where

$$
\begin{aligned}
g_{ \pm}(\zeta, \boldsymbol{q}, \Omega) & =\frac{e^{-\imath \Delta_{1}(\boldsymbol{q}, \Omega) \zeta} \pm e^{-\imath \Delta_{1}(-\boldsymbol{q},-\Omega) \zeta}}{2} \\
h_{ \pm}(\zeta, \boldsymbol{q}, \Omega) & =\frac{e^{-\imath D_{1}(\boldsymbol{q}, \Omega) \zeta} \pm e^{-\imath D_{2}(\boldsymbol{q}, \Omega) \zeta}}{2} .
\end{aligned}
$$

Eqs. (81)-(82) can be simplified making the following additional assumption

$$
\Delta_{1}(q, \Omega)=\Delta_{1}(q,-\Omega), D_{1}(q, \Omega)=D_{2}(q,-\Omega)
$$

This is always fulfilled for $q=\Omega=0$. In any case, we are mainly interested in the region arounf $q=0$ since, as in type I, image processing will be mainly efficient in this region. Inserting Eqs. (85) into Eqs.(81)-(82), the propagation equation for the field $a_{-}(\zeta, q)$ decouples leading to type I OPA equation with where $c_{S}(\zeta)$ plays the role of a $\zeta$-dependent pump

$$
\frac{\partial}{\partial \zeta} \hat{a}_{-}(\zeta, \boldsymbol{q}, \Omega)=c_{S}(\zeta) e^{-\imath \Delta_{1}(\boldsymbol{q}, \Omega) \zeta} \hat{a}_{-}^{\dagger}(\zeta,-\boldsymbol{q},-\Omega)
$$

The equations that describe the remaining two fields reduce to the equations of the type I SHG

$$
\begin{aligned}
\frac{\partial}{\partial \zeta} \hat{a}_{+}(\zeta, \boldsymbol{q}, \Omega)= & -c_{S}(\zeta) \hat{a}_{+}^{\dagger}(\zeta,-\boldsymbol{q},-\Omega) e^{-\imath \Delta_{1}(\boldsymbol{q}, \Omega) \zeta} \\
& -\sqrt{2} c_{+}^{*}(\zeta) \hat{a}_{S}(\zeta, \boldsymbol{q}, \Omega) e^{-\imath D_{1}(\boldsymbol{q}, \Omega) \zeta} \\
\frac{\partial}{\partial \zeta} \hat{a}_{+}(\zeta, \boldsymbol{q}, \Omega)= & +\sqrt{2} c_{+}(\zeta) \hat{a}_{+}(\zeta, \boldsymbol{q}, \Omega) e^{\imath D_{1}(\boldsymbol{q}, \Omega) \zeta}
\end{aligned}
$$

This decomposition of Type II SHG into a Type I SHG and a Type I OPA generalizes to the transverse spatial multimode case the conclusions obtained for a single spatial mode model in the traveling wave configuration [37], or in the cavity case [48].

To take advantage the coupling between waves with spatio-temporal frequencies $(\boldsymbol{q}, \Omega)$ and $(-\boldsymbol{q},-\Omega)$ generated by photon down conversion we consider a symmetrical input image as in subsection 3.2.

$$
\alpha_{ \pm}(q)=\alpha_{ \pm}(-q)
$$


The output intensity of the system depends now on the phase $\phi_{i n}$ of the input image. For simplicity we will consider an homogeneous phase for the input image: $\boldsymbol{\alpha} e^{\imath \phi_{i n}}$, where the elements of $\boldsymbol{\alpha}$ are real. The output intensity is then given by

$$
\left|\alpha_{i}^{\text {out }}(q)\right|^{2}=\left|\boldsymbol{U}_{i}(q, 0) \cdot \boldsymbol{\alpha}(q) e^{\imath \phi_{i n}}+\boldsymbol{V}_{i}(q, 0) \cdot \boldsymbol{\alpha}(q) e^{-\imath \phi_{i n}}\right|^{2} .
$$

The simplest detection scheme involves one detector located around $q$. The noise figure is then given by (70) with an extra factor 2 due to the symmetrization of the input image prior to injection.

$$
F_{i}=2 \frac{1+2 \boldsymbol{V}_{i}^{*}(q, 0) \cdot \boldsymbol{V}_{i}(q, 0)}{\left|\boldsymbol{U}_{i}(q, 0) \cdot \boldsymbol{\alpha} e^{\imath \phi_{i n}}+\boldsymbol{V}_{i}(q, 0) \cdot \boldsymbol{\alpha} e^{-\imath \phi_{i n}}\right|^{2} /\left|\alpha_{1}(q)\right|^{2}} .
$$

For OPA the noiseless character of image processing is only valid in the limit of large gains [19]. In the up-conversion case considered here the up conversion rate is never large. It is then crucial from the point of view of quantum imaging to consider the symmetrized detection scheme which involves two detectors located at opposite spatial points (corresponding to wave vectors in the telescopic configuration considered here). One measures the sum of the photocurrents:

$$
\hat{\mathcal{N}}_{i}(L, q)=\hat{N}_{i}(L, q)+\hat{N}_{i}(L,-q)
$$

The noise figure for this quantity is given by

$$
F_{i}=\frac{1+2 \boldsymbol{V}_{i}^{*}(q, 0) \cdot \boldsymbol{V}_{i}(q, 0)+2 R e\left(e^{-2 \imath \phi_{i}^{o u t}} \boldsymbol{U}_{i}(q, 0) \cdot \boldsymbol{V}_{i}(-q, 0)\right)}{\left|\boldsymbol{U}_{i}(q, 0) \cdot \boldsymbol{\alpha} e^{\imath \phi_{i} n}+\boldsymbol{V}_{i}(q, 0) \cdot \boldsymbol{\alpha} e^{-\imath \phi_{i n}}\right|^{2} /\left|\alpha_{1}(q)\right|^{2}}
$$

which differs from the one given in Eq.(70) by an additional interference term. $\phi_{i}^{\text {out }}$ denotes the phase of the output amplitude

$$
\phi_{i}^{\text {out }}=\operatorname{Arg}\left[\boldsymbol{U}_{i}(q, 0) \cdot \boldsymbol{\alpha} e^{\imath \phi_{i n}}+\boldsymbol{V}_{i}(q, 0) \cdot \boldsymbol{\alpha} e^{-\imath \phi_{i n}}\right] .
$$

For an OPA it has been shown that if the phase of the input image is adapted for maximal amplification, this amplification preserves the signal to noise ratio, and hence the operation is noiseless [41].

As previously stated, the phase sensitivity of the image processing scheme is an important requisite for interesting quantum imaging properties. This sensitivity is reflected in several quantities: First, the behavior of the OPA with a $z$-dependent pump can be characterized by the gain

$$
G_{\mathrm{OPA}}(q)=\frac{\left\langle N_{-}(L, q)\right\rangle}{\left\langle N_{-}(0, q)\right\rangle}
$$

Fig.15.a shows $G_{\mathrm{OPA}}(0)$ as a function of the phase of the input image. This gain oscillates between $\cosh (L)$ (maximal amplification) and $\operatorname{sech}(L)$ (minimum amplification). Second, the behavior of the Type I SHG, can be characterized by two quantities, the up-conversion and the transmission rate 

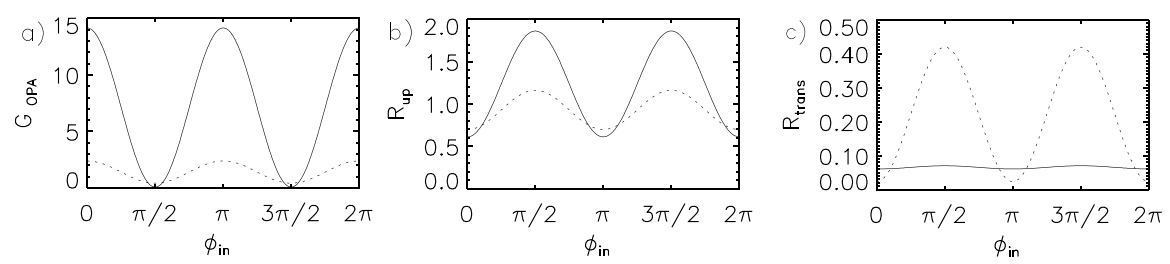

Fig. 15. Amplification factor (a), up-conversion rate(b) and transmission rate (c) as a function of the input phase for $\zeta=1$ (dotted line) and $\zeta=2$ (dashed line)

$$
R_{\mathrm{up}}(q)=\frac{\left\langle N_{S}(L, q)\right\rangle}{\left\langle N_{+}(0, q)\right\rangle}, R_{\mathrm{trans}}(q)=\frac{\left\langle N_{+}(L, q)\right\rangle}{\left\langle N_{+}(0, q)\right\rangle}
$$

The phase dependence of these quantities for $q=0$ is plotted in Fig. 15. In the limit of large propagation lengths, the up-conversion rate oscillates basically between a minimal value of $1 / 2$ and a maximum value of 2 which can be understood from the energy and phase conservation in SHG. The energy conservation implies that, after a complete conversion, the number of second harmonic photons has to be half the number of initial fundamental photons, and hence the amplitude is reduced by a factor $\sqrt{2}$. On the contrary, the phase of the output field is, after complete up-conversion, twice the phase of the input fundamental field. In the case of negligible phase mismatch, the maximal up-conversion rate occurs at $\phi_{i n}=\pi / 2$ and $3 \pi / 2$ for all distances. However, we should note that in general the phase for which the up-conversion is maximal may depend on the distance. Also, it is interesting to observe, in Fig. 15 that the input signal phase for which the up-conversion is minimal, ensures a maximal amplification of the - polarization component.

We consider now the noise behavior of the up-conversion process. We limit ourselves to the case of input phase adjusted for minimal/maximal upconversion and plot the up-conversion rate and the noise figure as a function of the propagation length (Fig. 16)

We observe that in the regime of minimal up-conversion, the noise figure rapidly drops to 1 , and hence image up-conversion is performed without noise addition. This can be understood because in the limit of large propagation lengths, the amplitude fluctuations entering the crystal at second harmonic frequency are damped during propagation, so that, in this limit they do not contribute to the noise in the amplitude of the second harmonic frequency output. This is not valid for the noise figure in the case of maximal upconversion. Defining $y_{i}(\zeta, q, \Omega)=\left(\hat{a}_{i}(\zeta, q, \Omega)-\hat{a}_{i}^{\dagger}(\zeta,-q,-\Omega)\right) / 2 \imath$, in the limit of large $\zeta$ one obtains

$$
y_{S}(z)=-\sqrt{2} y_{+}(0)+(1-z \tanh z) y_{S}(0)
$$

Therefore, the phase fluctuations of the input second harmonic field contribute to the total fluctuations and the noise figure is larger than 1, as shown in Fig. . Only for propagation lengths such that 

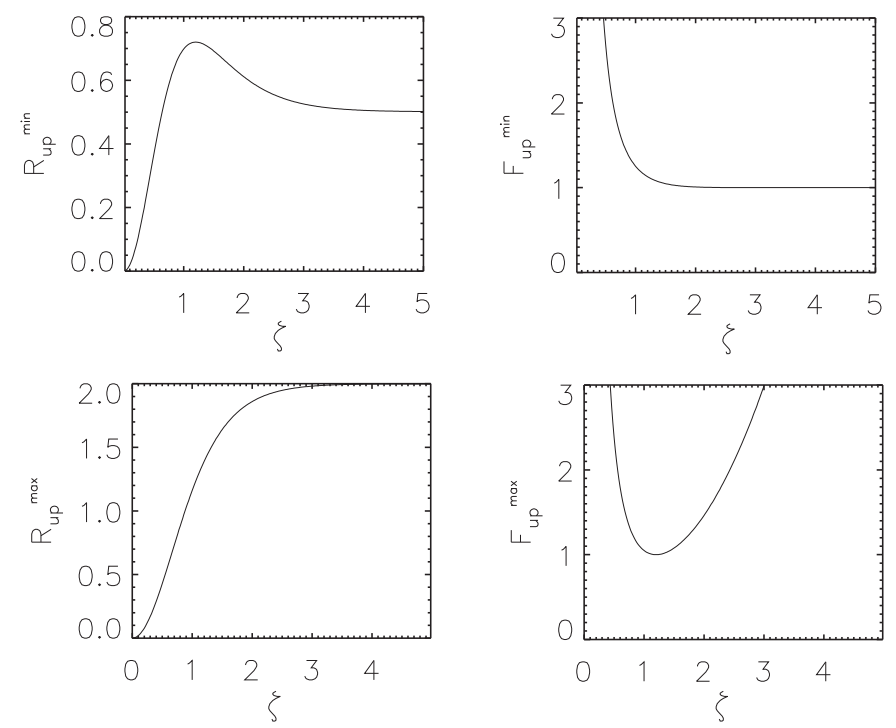

Fig. 16. Up-conversion rate and Noise Figure at a vanishing transverse wave vector $q=0$ as a function of the propagation length in the symmetric detection scheme. Top row $\phi_{i n}=0$, bottom row $\phi_{i n}=\pi / 2$

$$
1-\zeta \tanh (\zeta)=0
$$

the second harmonic input fluctuations do not contribute and the image processing is noiseless $(F=1)$

Finally, we consider the behavior of this image processing scheme as a function of the position in the transverse plane. Fig. 17 shows the upconversion rate and the OPA gain for an input phase corresponding to minimum up-conversion (maximum OPA gain). The corresponding noise figures for the single detector placed at $q$ and the symmetric detector scheme with two detectors at $q$ and $-q$ are shown as well. The up-conversion rate and the OPA gain are roughly constant on a disk of finite diameter centered on the main optical axis of the system $(q=0)$. The noise figure of the up-conversion $F_{u p}$ rate approaches in this central region the value of 1 characterizing a noiseless image processing. However, this interesting property is only true in the symmetrical detection scheme. If the output would be detected only with one detector, a large amount of excess noise with respect to the noise in the injected image would be observed. For the OPA gain, in the central region of maximal gain, the two detection scheme give exactly the same noise level, as a consequence of the strong nonclassical correlations of the two outputs at $q$ and $-q$. At higher $q$ where almost no amplification takes place, the noise figure $F_{\mathrm{OPA}}$ for the symmetric and single detector case differ by a factor 2 as a consequence of the symmetrization procedure of the input image. 

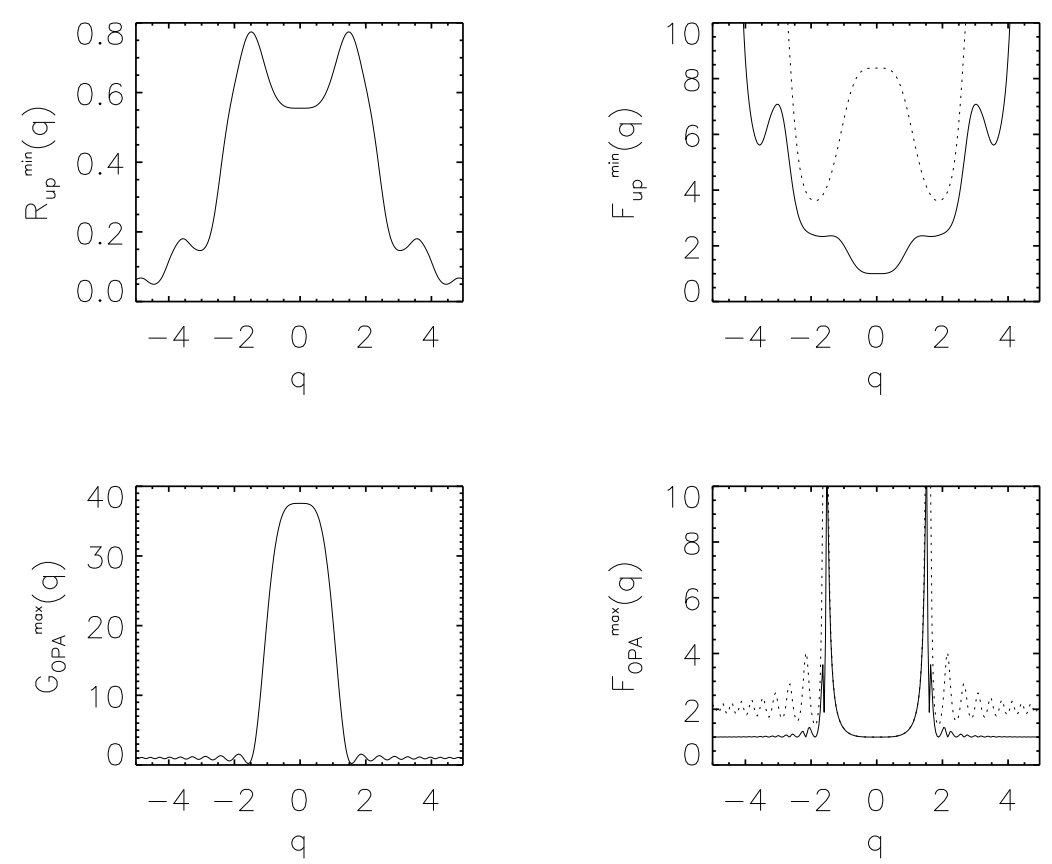

Fig. 17. Up-conversion rate (top left) and OPA gain (bottom left) as function of the position in the transverse plane for $\phi_{i n}=0$. The corresponding noise figures are shown on the right. The dotted line corresponds to the single detector case while the solid line corresponds to symmetric detection.

\section{References}

1. J. Teuber, Digital Image Processing, Prentice Hall, 1993.

2. J. Mitwinter and J. Warner, J. Appl. Phys. 38 (1967) 519

3. J. Mitwinter, Appl. Phys. Lett. 12 (1968) 68

4. F. Devaux, A. Mosset, E. Lantz, S. Monneret and H. Le Gall,Appl.Opt.40 (2001) 4957

5. A.H. Firester, J. Appl. Opt. 40, 4842 (1969); 4849 (1969)

6. F. Devaux and E. Lantz, J. Opt. Soc. Am. B 12 (1995) 2245

7. E. Lantz and F. Devaux, Quantum Semiclass. Opt. 9 (1997)279

8. F. Devaux and E. Lantz, Opt. Commun. 114 (1995) 295

9. P. Günther, J. P. Huignard eds. Photorefractive Materials and Their Applications II, Topics in Applied Physics Vol. 62. Springer-Verlag, New York 1989

10. J. Feinberg, Opt. Lett. 5 (1980) 330

11. J.P. Huignard, J. P. Herriau, Appl. Opt. 17 (1978) 2671

12. B. Liang, Z. Wang, J. Guang, G. Mu, C. M. Cartwrigt Opt.Lett.25 (2000) 1086

13. E. Ochoa, L. Hesselink, W. Goodman, Appl. Opt. 24 (1985)1826 
14. Y. H. Ja, Opt. Comm. 44 (1982) 24

15. Y. H. Ja, Opt. Quant. El. 15 (1983) 457

16. Y. H. Ja, Appl. Phys. B 36 (1985) 21

17. H. Rajbenbach, A. Delboublé, J. P. Huignard Opt. Lett. 14(1989) 1275

18. M. D. Rahn, D. P. West, J. D. Shakos, J. Appl. Phys. 87(2000) 127

19. A. Gatti, E. Brambilla, L. A. Lugiato and M. I. Kolovob, J. Opt. B: Quantum Semiclassical Opt. 2, 196 (2000)

20. European Physical Journal, special issue D 22

21. C. M. Cave, Phys. Rev. D 26 (1982) 1817.

22. Z. Y. Ou, S. F. Pereira, H. J. Kimble, Phys. Rev. Lett. 70 (1993) 3239.

23. J. A. Levenson, I. Abram, Th. Rivera, Ph. Grangier, J. Opt. Soc. Am. B 10 (1993) 2233.

24. M.I. Kolovob and I. V. Sokolov, Zh. Ékps. Theor. Fiz. 96, 1945 (1989) [Sov. Phys. JETP 69, 1097 (1989)], Phys. Lett. A

25. M. I. Kolobov, Rev. Mod. Phys. 71, 1539 (1999)

26. S. K. Choi, M. Vasilyev, P. Kumar, Phys. Rev. Lett. 83, (1999) 1938

27. P. Scotto, P. Colet, M. San Miguel, Optics Letters 28, 1695 (2003)

28. U.Peschel, C. Etrich, F.Lederer, Opt. Lett, 23, 500 (1998)

29. U.Peschel, C. Etrich, F.Lederer, Phys. Rev E, 58, 4005 (1998)

30. S. Longhi, Opt. Lett, 23, 346 (1998)

31. S. Longhi, Phys. Rev. A, 59, 346 (1999)

32. Z. Y. Ou, Phys. Rev. A. 59, 4021 (1999)

33. A. Jacobo, P. Colet, P. Scotto and M. San Miguel, submitted for publication.

34. P. Scotto and M. San Miguel. Phys. Rev. A 65, 043811 (2002)

35. C.L. Tang and L.K. Fundamentals of Optical Parametric Processes and Oscilators, Laser Science and Techology, Vol. 20 (Harwood Academic, Amsterdam, 1995), pp. 30 and 31.

36. R. D. Li and P. Kumar, Phys. Rev. A 49, 2157 (1994)

37. Z.Y. Ou, Phys. Rev. A 49, 2106 (1994)

38. M.K. Olsen, R. J. Horowicz, L. I. Plimak, N. Treps. and C. Fabre, Phys. Rev. A. 61, $021803(2000)$

39. M.K. Olsen, R. J. Horowicz, Opt. Commun. 168, 135 (1999)

40. M.K. Olsen, L. I. Plimak, M.J. Collet, and D.F. Walls, Phys. Rev. A. 62, $023802(2000)$

41. M.I. Kolobov and L.A. Lugiato, Phys. Rev. A 52, 4930 (1995)

42. I. V. Sokolov, M. I. Kolobov and L. A. Lugiato, Phys. Rev. A. 60, 2420 (1999)

43. A. Berzanskis, W. Chinaglia, L.A. Lugiato, K.-H. Feller and P. Di Trapani, Phys. Rev. A 601626 (1999).

44. E. Lantz and F. Devaux, Quantum Semiclassic. Opt. 9, 279 (1997)

45. C. M. Caves, Phys. Rev. D 26, 1817 (1982)

46. P. Scotto, Phys. Rev. A 68, 033814(2003)

47. J. A. Armstrong, N. Bloembergen, J. Ducuing and P. S. Pershan, Phys. Rev. 127, 1918 (1962)

48. Z.Y. Ou, Phys. Rev. A 49, 4902 (1994) 